\title{
HACIA UN PLANTEAMIENTO DE ARQUITECTURA DOCENTE, EN CHILE
}

\author{
(TOWARDS A TEACHING ARCHITECTURE IN CHILE)
}

\author{
Oscar Mac Clure Alamos, Arquitecto
}

Universidad Católica de Chile

\section{RESUMEN}

En estas páginas se pretende dar a conocer diferentes soluciones arquitectónicas adoptadas en los proyectos escolares, a través de mi experiencia profesional, durante 22 años (1959 - 1981) en la Sociedad Constructora de Establecimientos Educacionales en Santiago de Chile.

También la ejecución, durante 4 años (1981 - 1985), en la Universidad de Santiago (ex Universidad Técnica del Estado) del proyecto modificado y la construcción del edificio para el Planetario, que había realizado en la Sociedad Constructora.

En forma breve expondré, además, algunas obras de carácter educacional, realizadas en el ejercicio privado de mi profesión.

En base a estas experiencias procuraré entregar algunas sugerencias y conclusiones que podrian ser útiles en futuros programas destinados a resolver los problemas de las construcciones docentes.

Desde una posición critica y objetiva intentaré también dar a conocer la situación actual de la arquitectura educacional en el pais.

\section{SUMMARY}

On this pages the author expound the different architectural solutions adopted for school projects from his professional experience: 22 years (1959-1981) in the Construction Society of Educational Establishments in Santiago de Chile, as well as the execution -four years at the Santiago University (late State Technical University)- of the modified project for the

Planetarium which had been projected by the Construction Society.

He also expound briefly a number of constructions of educational character carried out through the practising of his profession.

Taking as a base these experiences, he offers some suggestions and conclusions that will be useful for future programmes intended to solve the problems of the school buildings.

From a critical and objective position he tries to show the actual situation on the educational architecture in this country.

\section{INTRODUCCION}

En la República de Chile, desde los albores de su Independencia, la educación fue tarea prioritaria del Estado. Don Andrés Bello (1781-1865) primer Rector de la Universidad de Chile, pronuncia el discurso inaugural el 17 de septiembre de 1843 y enfatiza: "yo ciertamente soy de los que miran la instrucción general, la educación del pueblo, como uno de los objetos más importantes y privilegiados a que pueda dirigir su atención el Gobierno; como una primera y urgente; como la base de todo sólido progreso; como el cimiento indispensable de las instituciones republicanas". Señala más adelante: “La Ley, al plantear de nuevo la Universidad (...) ha dado a una de las secciones del cuerpo universitario el encargo especial de velar sobre la instrucción primaria, de observar su marcha, de fa- cilitar su propagación, de contribuir a sus progresos".

Bello planteó también la necesidad de establecer Escuelas Normales para preceptores; Manuel Montt y Domingo Faustino Sarmiento serán los realizadores.

Montt, maestro, jurisconsulto, político y Presidente reelecto en el periodo 1851-1861 supo captar la vocación por la enseñanza que tenia Sarmiento y lo vio como el maestro capaz de llevar a cabo la tarea.

Asi, en 1842 es fundada la primera Escuela Normal de América, que es realización del llustre exiliado Argentino, gran educador y, más tarde, Presidente de la República de Argentina.

\section{NOTA}

Se respetan, en todos los artículos de este numero de Informes, palabras empleadas por los autores de los distintos trabajos que, aún siendo totalmente castellanas, son poco usuales en España. 
El desenvolvimiento de la enseñanza fue una constante progresiva. Las experiencias que traen al pais destacados científicos y humanistas extranjeros, contratados por los sucesivos Gobiernos, representan un valioso aporte, especialmente en los periodos iniciales del magisterio chileno.

La rápida evolución de la Ciencia y Tecnologia contemporánea y su consecuente repercusión en las Ciencias de la Educación, originan en el pais la necesidad de realizar profundos cambios en el ámbito educacional. Se generan, entonces, sucesivas reformas que pretenden dar soluciones adecuadas a los cambios culturales y sociales del pais.

Frente a esta situación, surgió la necesidad de dar una respuesta arquitectónica conformada a los nuevos requerimientos educacionales. La obra arquitectónica debia, en cierta medida, flexibilizarse, hacerse más dinámica para dar un marco adecuado a las inquietudes renovadoras de la docencia.

Los esfuerzos desplegados por los Gobiernos, en materia de construcciones escolares, se canalizaron en el año 1937 -durante el Gobierno del Presidente, don Arturo Alessandri P.-, en la creación de la Sociedad Constructora de Establecimientos Educacionales. Este Organismo, dependiente del Ministerio de Educación, al que también se aportaron capitales privados desde su fundación, tuvo atribuciones autónomas y suficientemente descentralizadas como para desarrollar con gran agilidad la ejecución de planes educacionales anuales. Estos planes involucraban proyectos y construcciones para la Enseñanza Básica y Media, como también de Escuelas Técnicas, Agrícolas, Industriales, Normales e Institutos Comerciales. Abordó, además, otro tipo de proyectos especiales relacionados con la actividad cultural como: Museos, Aulas Magnas, Centros de Perfeccionamiento para el Magisterio, Centros Artísticos, Planetarios, etc.

La finalidad de los planes gubernamentales era equipar escolarmente el país, en todos los estamentos educacionales ya señalados. Estos propósitos se irán concretando en la ejecución de edificios escolares.

A continuación se exponen algunos proyectos experimentales realizados por el Taller de Arquitectura de la Sociedad Constructora de Establecimientos Educacionales, los cuales se hallan, directa o indirectamente, clasificados en base a sistemas constructivos y ordenados en forma cronológica.

En esta exposición se mencionan también proyectos realizados en esta Sociedad Constructora por otros Arquitectos o equipos inter- disciplinarios, en los que personalmente no participé, pero que considero interesantes aportes a la especialidad de la arquitectura escolar. Se ha señalado además, en el presente trabajo, algunas obras de carácter privado que se refieren a la actividad docente. Por razones obvias, se han excluido expresamente los planteles superiores de indole universitarios.

\section{PROYECTOS EXPERIMENTALES REALIZADOS}

\subsection{Albañilería reforzada de bloques de cemento: Sistema atípico}

El primer trabajo que se me encomendó, en 1959, fue el proyecto, ejecutado ese mismo año, de la Escuela Básica Barrio Norte de Antofagasta (Lámina I), en el que se planteó un sistema constructivo de hormigón armado modulado, en pabellones separados, con caracteristicas propias del lugar y adecuadas al clima desértico del Norte del pais. La albañilería reforzada se consultó en bloques de cemento premoldeados, material existente en el lugar. Para esta Escuela se diseñó un sistema de protección solar basado en elementos prefabricados in situ de hormigón armado en placas premoldeadas de $1,00 \times 3,00 \mathrm{~m}$, bloques huecos de cemento para ventilación transversal y sombreaderos. Más adelante, vinieron otros proyectos que tuve que desarrollar, entre los cuales está la Escuela Técnica de Antofagasta.

La mencionada Escuela no pudo realizarse, como se habia concebido, ya que el proyecto fue rechazado por no estar entonces encuadrado dentro de los cánones tradicionales de la Sociedad.

Este contemplaba un bloque principal de cuatro pisos frente al mar, y una placa de dos pisos destinada a administración y zona de esparcimiento.

Debido a la escasez de terreno se proponia utilizar la terraza del segundo volumen como patio de juegos, unido por un puente al edificio principal. Todo el conjunto estaba diseñado en hormigón armado, con losas nervadas perfectamente moduladas. Con posterioridad, entre los años 1961 y 1964 , la Junta de Adelanto de Arica, en el extremo Norte del pais, inició una politica de renovación pedagógica.

Entre los edificios que se ejecutaron, conforme a la nueva modalidad educativa, se pueden mencionar mis proyectos del Colegio Medio de Niñas y Colegio Medio de Hombres de Arica, en los cuales se siguieron utilizando técnicas tradicionales de hormigón armado, con la aplicación de elementos premoldeados de protección solar (Lámina II). 


\section{Lámina I}

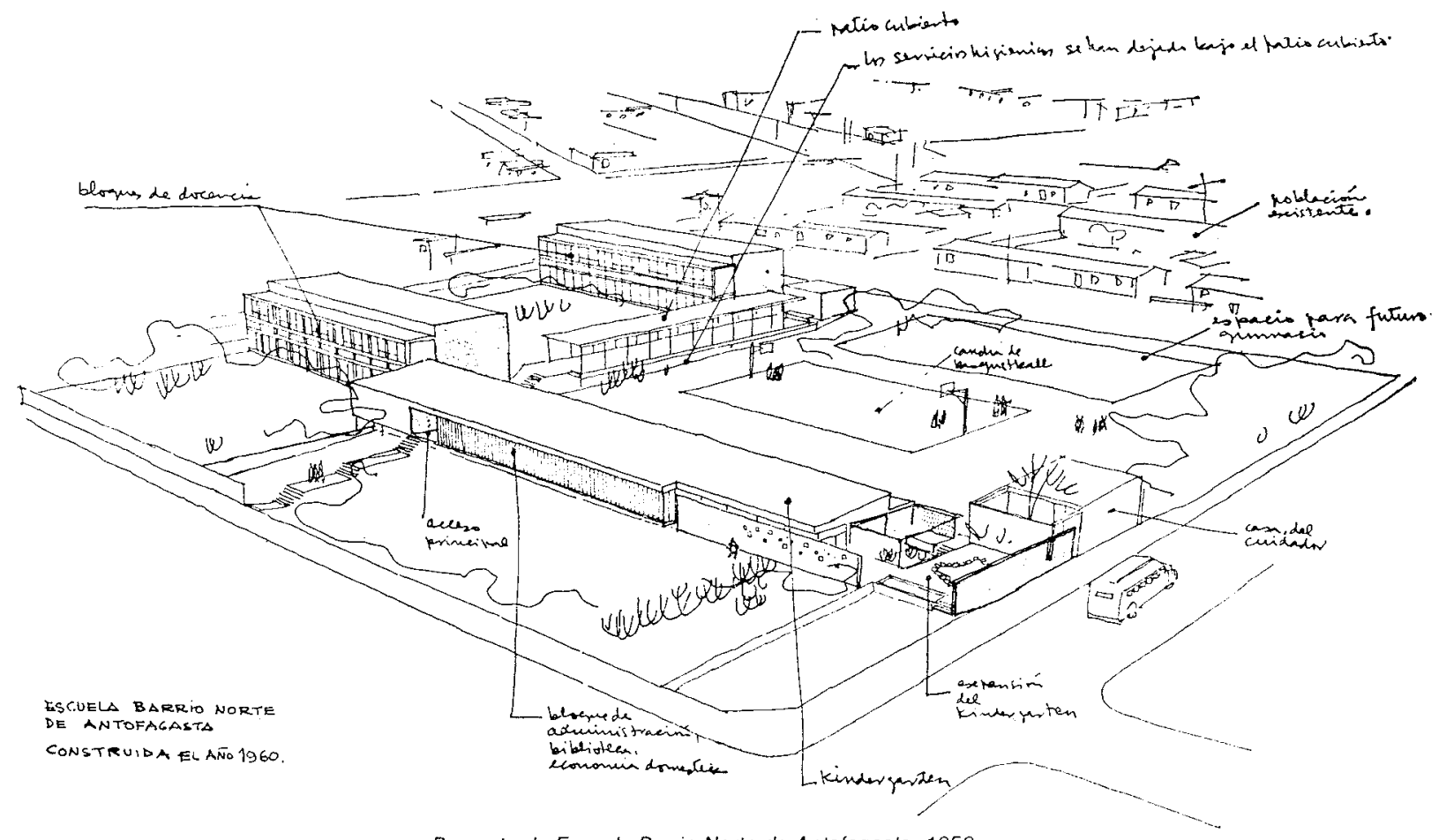

Proyecto de Escuela Barrio Norte de Antotagasta, 1959.

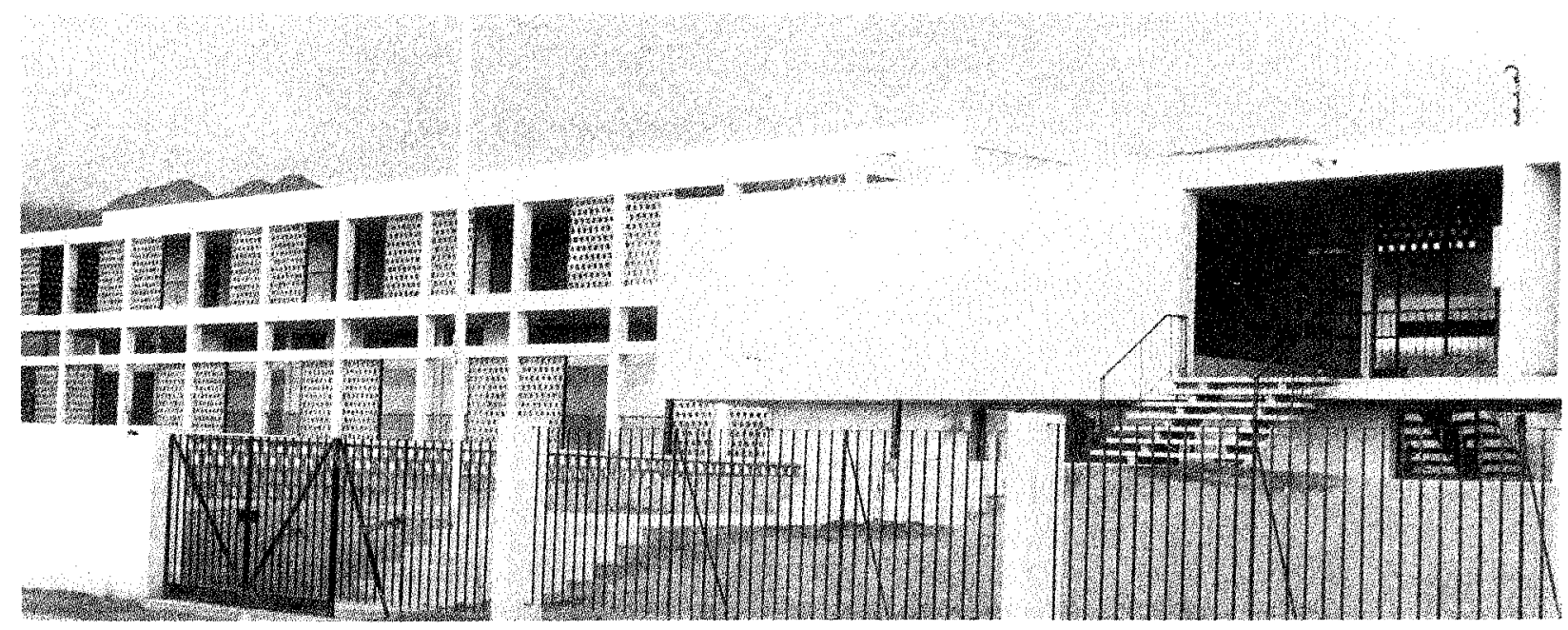

Escuela Barrio Norte de Antofagasta. Acceso principal

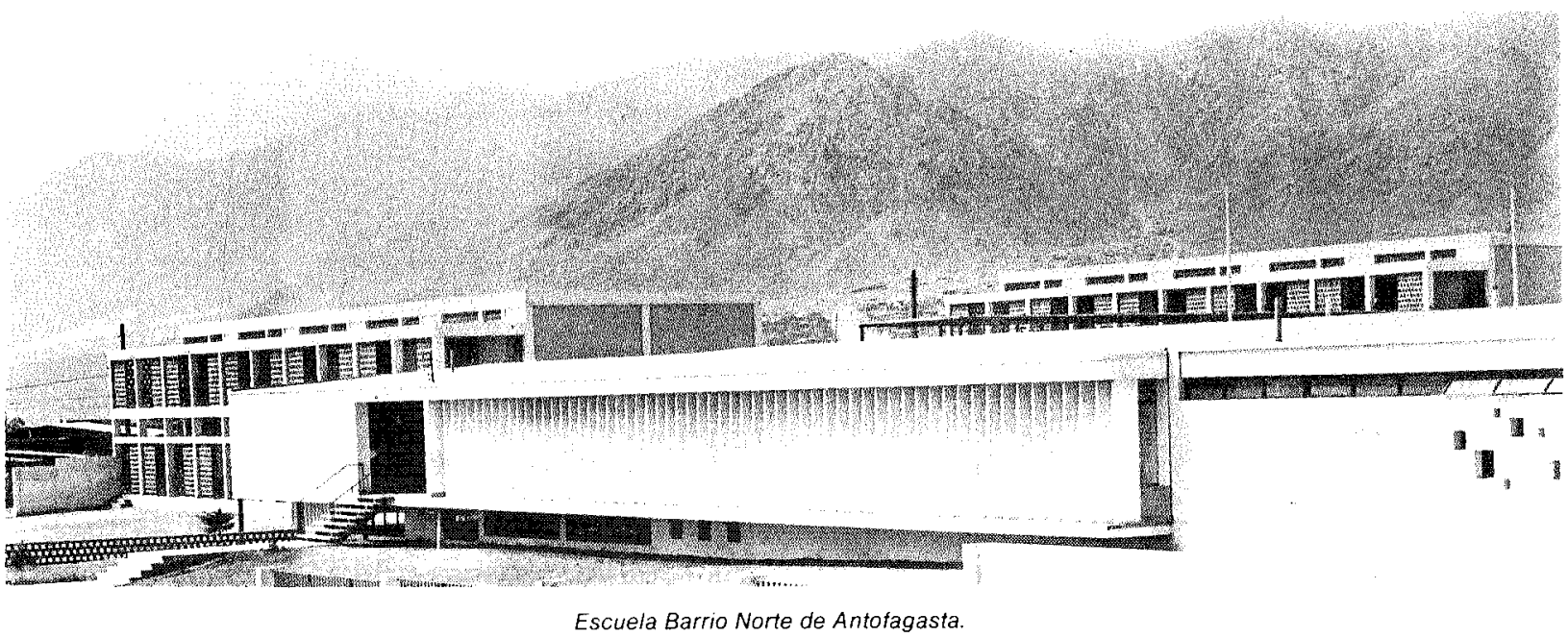




\section{Lámina II}
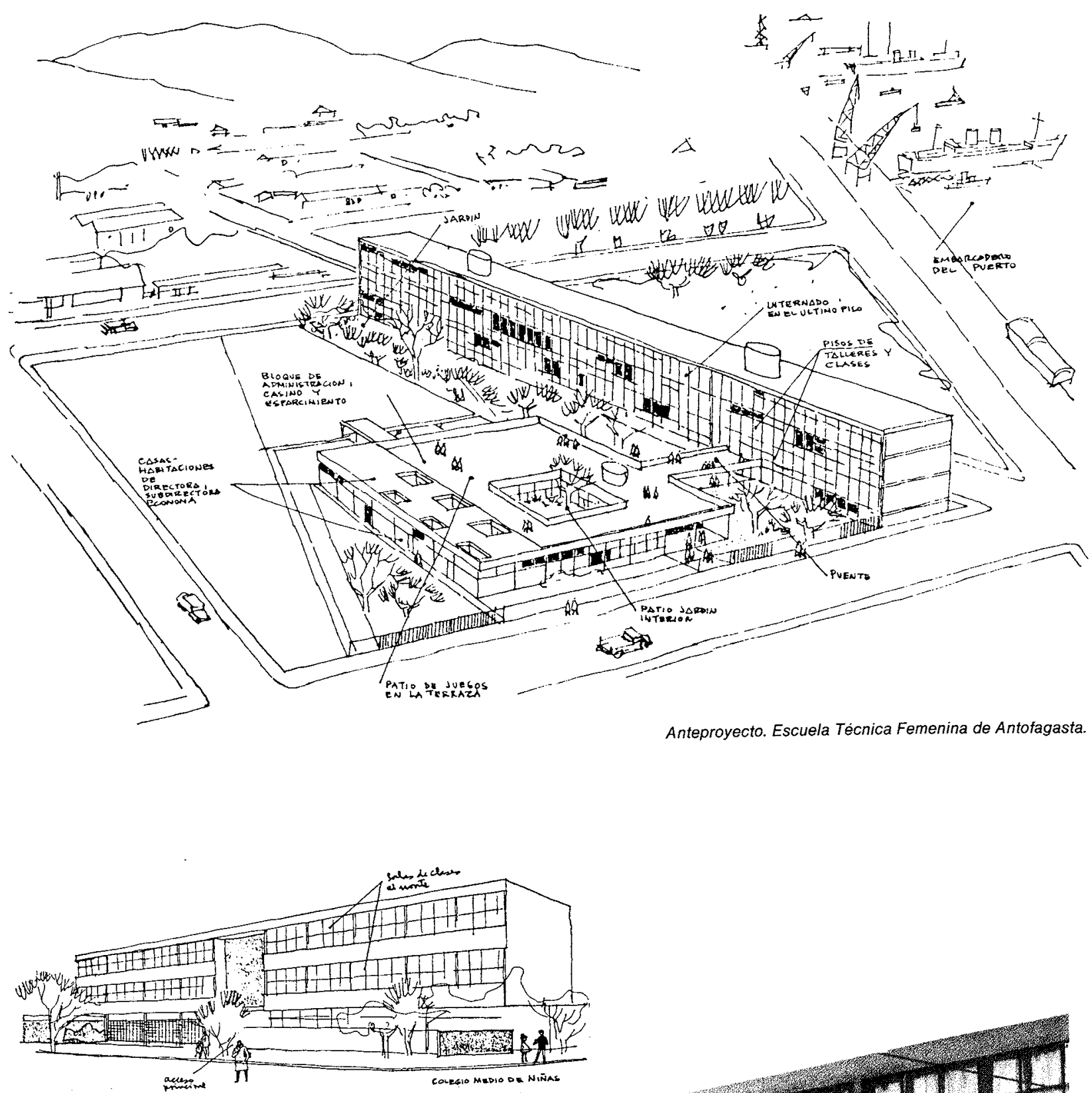

Colegio Medio de Ninas de Arica, 1962 


\subsection{Hormigón armado estructural: Sistema atípico}

En esta categoria podria mencionar la Escuela Técnica Femenina de Concepción, cuyo proyecto ejecuté en 1963 y que constaba de salas de clases e internado, en dos y tres plantas (Lámina III).

En el año 1964, la Sociedad Constructora me encargó el edificio para el Instituto Comercial de San Fernando, en el que se utilizaron experimentalmente losas nervadas de hormigón armado apoyadas en vigas longitudinales, las cuales descansaban en pilares metálicos y en los indispensables muros de arrostramientos sismicos.

Este sistema permitió el uso de paneles divisorios removibles en la separación de espacios docentes, a fin de adaptarlos a distintas situaciones para las nuevas exigencias pedagógicas (Lámina III).

\subsection{Hormigón armado modulado: Sistemas tipificados}

Descartados los sistemas constructivos tradicionales por el lento desarrollo en su planificación y ejecución en obra, se recurrió a la utilización de un sistema de tipificación de estructuras modulares, basado en marcos rigidos, pilares, vigas y losas de hormigón armado.

Uno de ellos -denominado Tipo 501- proyecto realizado en 1965, sirvió de modelo estructural repetitivo para la construcción de innumerables edificios a lo largo de todo el pais, en áreas urbanas, durante un extenso periodo de vigencia de planes anuales normales y extraordinarios. El módulo de polarización y de viguetas se fijó en $2,40 \mathrm{~m}$, entre ejes, salvando una luz constante de $6,00 \mathrm{~m}$, en simple crujia, con corredores cubiertos según la extensión de cada bloque.

Como ejemplo del mismo se puede mencionar e que apliqué en el proyecto realizado del Liceo n.o 1 de Niñas de Viña del Mar.

En el año 1968, se entregaron otros edificios para la Enseñanza Media, como los Liceos n.o 4 de Recoleta y n.o 17 de Las Condes de Santiago, donde tuve la oportunidad de aplicar un nuevo prototipo llamado Tipo 505, en reemplazo del anterior ya descrito, el cual fue elaborado por un equipo interdisciplinario del Sub-Departamento de Investigaciones a mi cargo (Lámina IV).

El objetivo de este proyecto Tipo fue el de perfeccionar el sistema anterior, pero manteniendo la misma estructura de marcos rígidos a 2,40 m, entre ejes, e incorporando la presencia de la albañileria de ladrillo prensado a la vista en forma sobrepuesta a los pilares de hormigón armado.
Aquello representa un intento formal de enriquecimiento exterior del edificio escolar. Dicho prototipo se utilizó en la mayor parte de los edificios de Enseñanza Superior y Especializada, logrando una gran flexibilidad de los espacios aéreos interiores y evidente economia de costos de mantenimiento (Lámina V).

\subsection{Estructura metálica modulada: Sistemas tipificados}

Con objeto de dar una respuesta a breve plazo, y en forma cada vez más acelerada, a los requerimientos para resolver la demanda de establecimientos educacionales, se propuso proyectar y emplear un nuevo sistema estructural metálico, basado en experiencias ya realizadas a gran escala en México.

Dicho proyecto -llamado MC en un piso, se fue paulatinamente perfeccionando y modificando para adecuarlo a los materiales regionales y a las condiciones económicas del país. La última versión -Tipo $401 \mathrm{~F}$ - consistió en pilares y vigas de planchas dobladas de $4 \mathrm{~mm}$, de acero, con los cuales se formaban marcos rígidos en ambos sentidos. La estructura de techumbre estaba constituida por paneles de madera prefabricados en obra, con cubiertas de planchas de asbestocemento ondulado, o de fierro galvanizado (Lámina VI).

Los muros exteriores (testeros, longitudinales, antepechos) podian ser de madera, ladrillo, piedra, paneles prefabricados (opacos o vidriados) dependiendo del uso de éstos, según los materiales, mano de obra y climas regionales.

El módulo empleado fue de $3,00 \mathrm{~m}$, entre ejes de pilares metálicos estructurales y obtuvo grandes ventajas por su versatilidad y facilidad de transporte, pudiendo ser armados sus elementos constitutivo's, mediante pernos, en sitios alejados y de difícil acceso. Generalmente fue destinado a resolver las Escuelas Básicas rurales, casi en todo el territorio nacional. Como ejemplo de ello puedo citar la Escuela Básica n.o 13 - Gabriela Mistral, que proyecté y se ejecutó en 1970 en la localidad de Monte Grande, en el Valle de Elqui, interior de la precordillera, en el Norte de Chile.

La eficacia del sistema permitió que se iniciaran gestiones de exportación de aulas a paises limitrofes. Asimismo, fueron donados por el Gobierno nuestro, Centros Educacionales a Perú y Colombia, utilizando esas estructuras fabricadas en el pais, como simbolos de acercamiento e integración americanos (Lámina VII). 


\section{Lámina III}

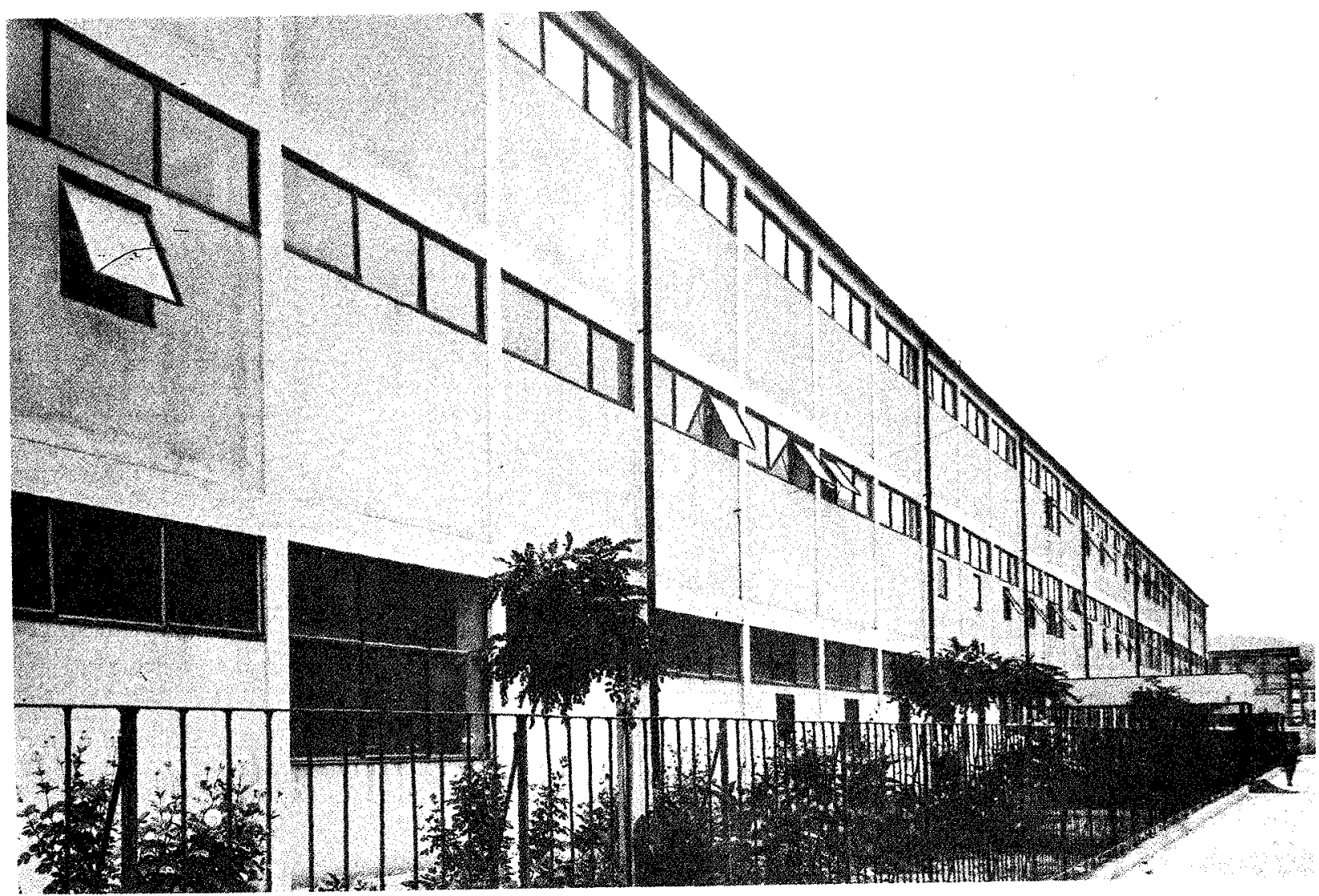

Escuela Técnica Femenina de Concepcion. 1963.

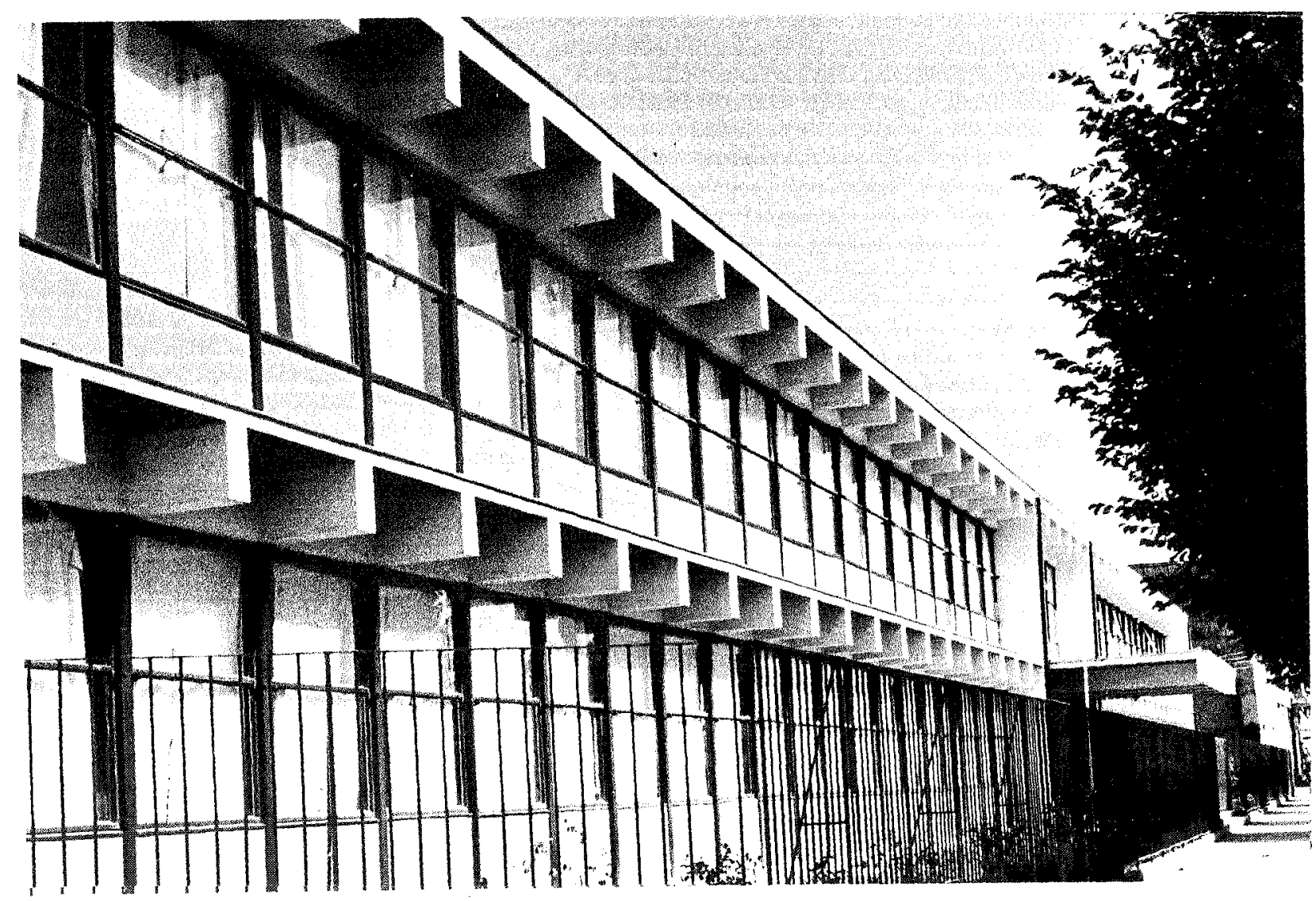

Instituto Comercial de San Fernando, 1964. 


\section{Lámina IV}

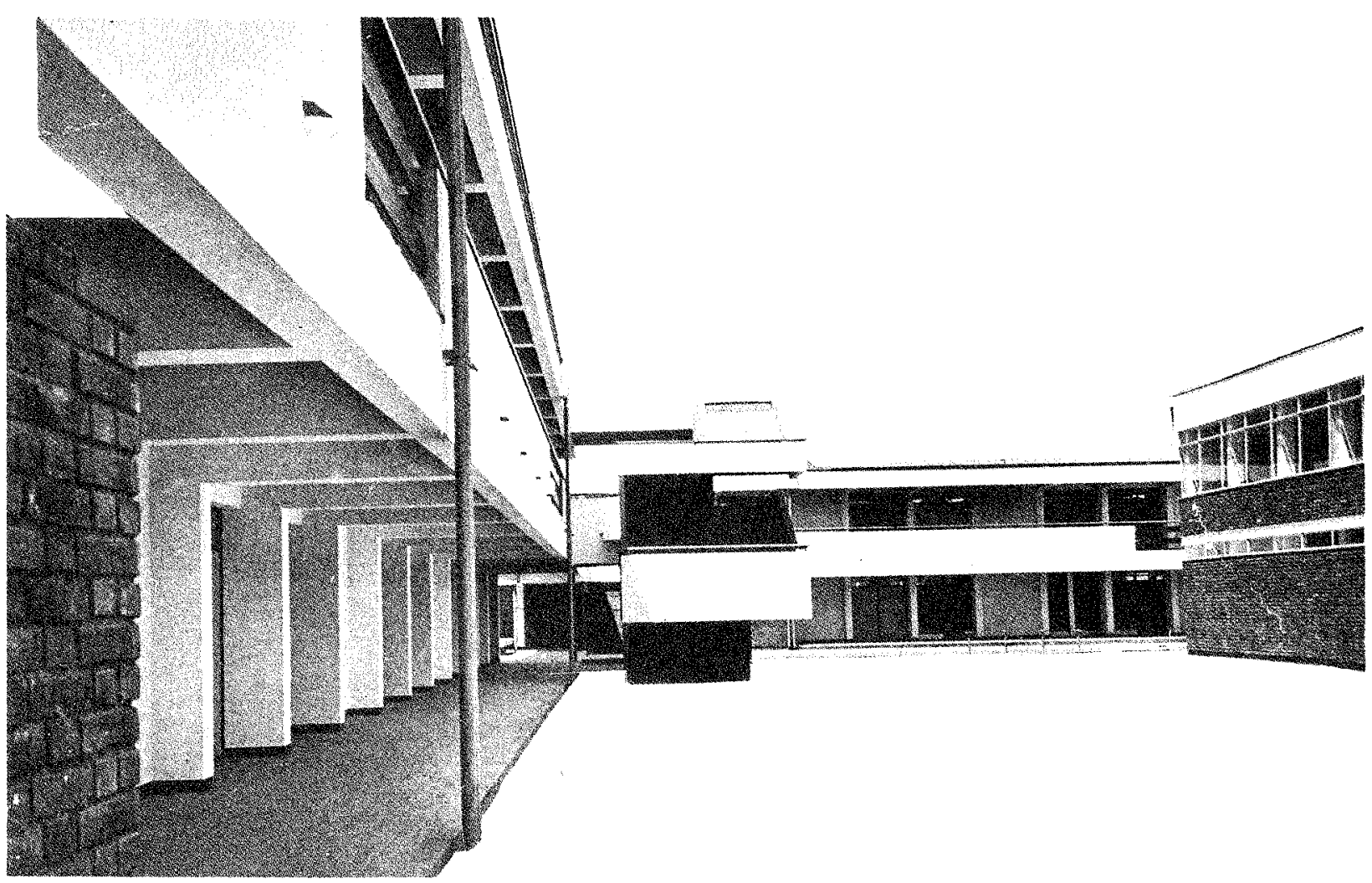

Liceo n.0 4, Recoleta de Santiago, 1968

\section{Lámina V}

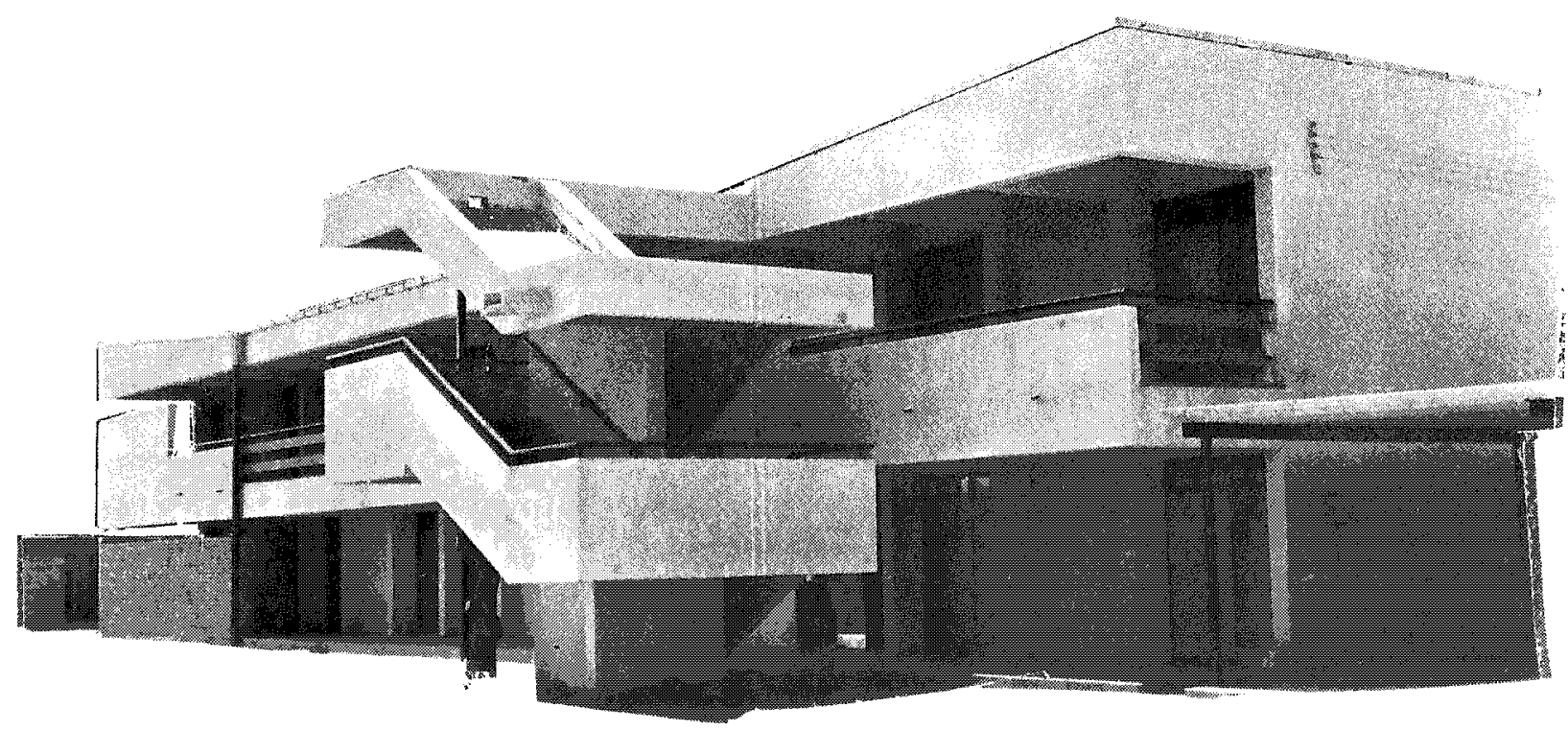

Liceo n.o 17, Las Condes de Santiago, 1968 
16

Informes de la Construcción, Vol. 38, n. 386 , diciembre, 1986
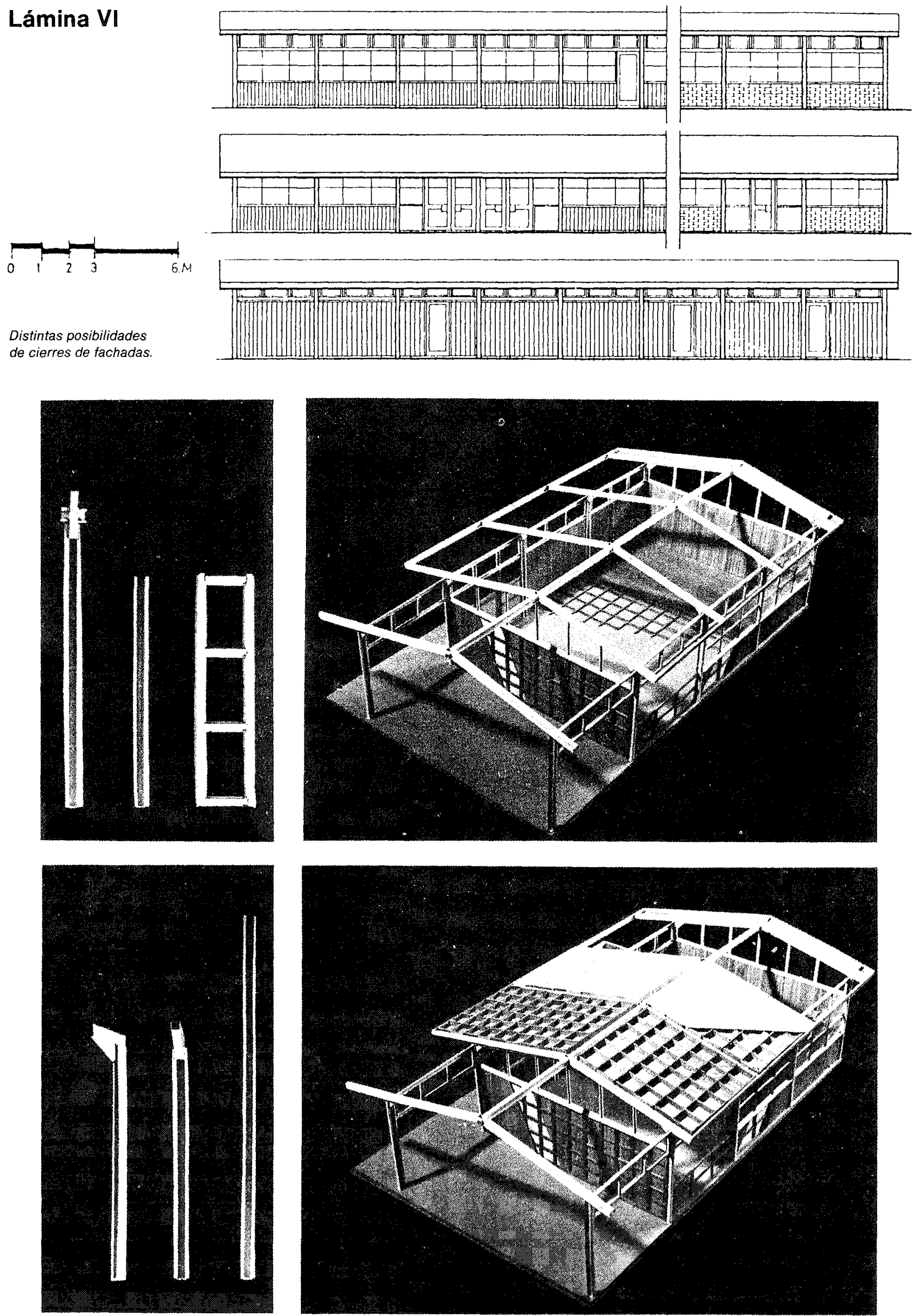

Proyecto tipo MC 401 - F. Estructura 


\section{Lámina VII}

Escuelas tipo MC $401-F$.

donadas por el Gobierno chileno, 1971

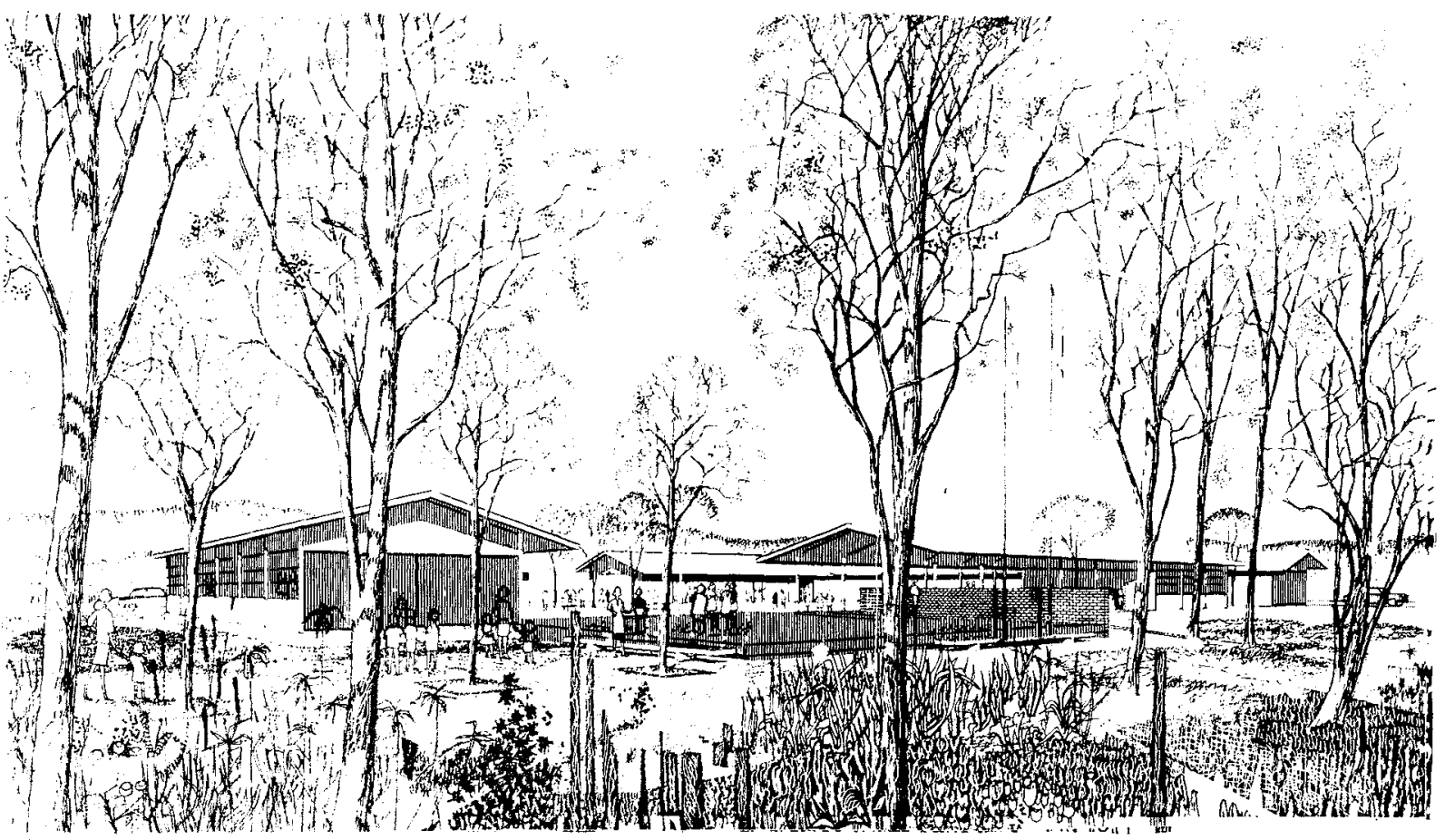

Escuela donada a la República del Perú y construida por estudiantes chilenos, 1971

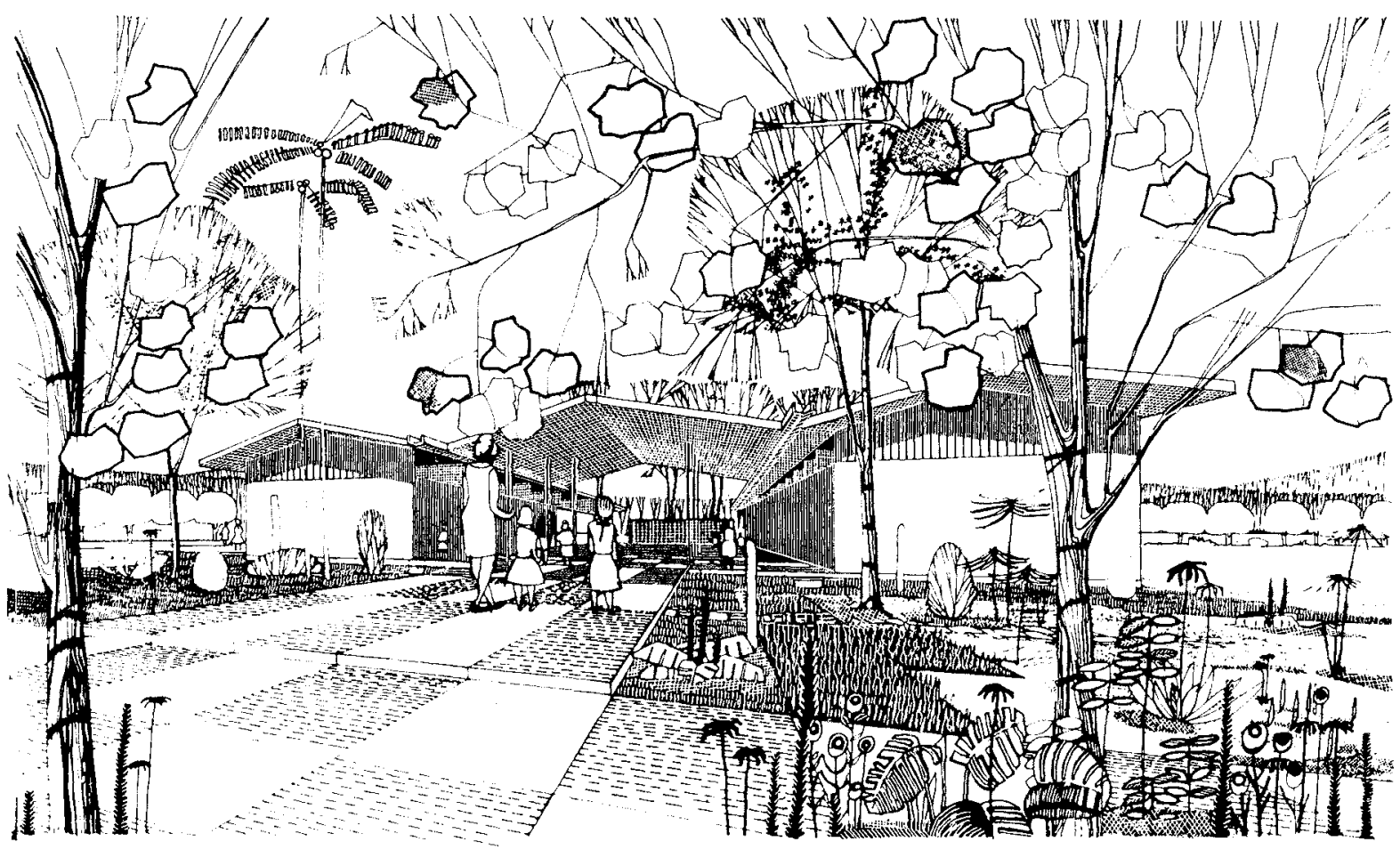

Centro Educacional en la Poblacion Eduardo Frei, Bogota. 


\subsection{Estructura mixta modulada: Sistemas tipificados}

A fin de obtener una mayor densidad de edificación en sectores urbanos, de precios más limitados, se planteó la necesidad de contar con un proyecto tipificado en dos plantas.

De alli que se desarrolló en el año 1967 un proyecto Tipo $606-F$, que era una combinación de un proyecto tradicional en hormigón armado en primer piso y el proyecto $401-F$, ya descrito en el segundo piso. Los pilares y vigas de la planta baja coinciden en sus ejes transversales de $6,00 \mathrm{~m}, \mathrm{y}$ en los longitudinales de $3,00 \mathrm{~m}$, con la estructura metálica sobrepuesta (Lámina VIII). En el año 1967, cuando ya se habian iniciado diversos estudios de nuevas soluciones arquitectónicas en el Taller de investigaciones a mi cargo, se desarrolló una versión similar al tipo 606, que se denominó n.० 720 , que combinaba como el anterior, en forma mixta, una estructura de hormigón armado en primer piso y una metálica en planta alta. Su diseño difería del otro en el sentido de que se contemplaba un sub-módulobase de $1,20 \times 1,20 \mathrm{~m}$, con pilares a $3,60 \mathrm{~m}$ entre ejes y una luz interior de $7,20 \mathrm{~m}$. El proyecto que se aplicó por primera vez en la Escuela Básica n.o 87, Lord Cochrane, en Santiago, de 4.000 $\mathrm{m}^{2}$ de superficie, contemplaba una estructura anexa de acceso, de carácter experimental, integramente metálica, que permitia un uso bidireccional del espacio interior. El mencionado proyecto se empleó en diferentes obras. Este edificio, con caracteristicas especiales, sirvió para la utilización e investigación de nuevas técnicas de moldajes o encofrados para hormigones vistos, escaleras semi prefabricadas metálicas, paneles divisorios desmontables, etc.

\subsection{Estructura modulada de madera: Sistema tipificado}

En el mismo año 1969, la Sociedad Constructora se habia propuesto diseñar alternativas en madera, para solucionar los edificios en el Sur del pais, donde abunda ese material. Un equipo de arquitectos, que reunió los antecedentes técnicos necesarios y el conocimiento previo de los terrenos disponibles, donde debian emplazarse dos establecimientos educacionales, programados para la ciudad de Puerto Montt, propuso la utilización del proyecto experimental Tipo 801, en madera.

Me ha parecido importante mencionar la aplicación de este sistema constructivo, elaborado en el taller de Arquitectura de la Zona Sur de la Sociedad Constructora, en consideración a que permitió lograr una certera adecuación al lugar, de un conjunto educacional.
Los desniveles topográficos se abordaron con unidades modulares independientes que podian conectarse, entre sí, a nivel o en desnivel, sin necesidad de agregar un elemento separador o relacionador. Dichas unidades, ejecutadas en estructuras de madera y elementos de apoyo metálicos, fueron concebidas con el objeto de obtener la máxima adaptación a los cambios pedagógicos, creando espacios integrales interiores. En este subtitulo, relacionado con construcciones escolares en madera, se puede mencionar la iniciativa del Ministerio de Educación para la realización de Aulas Provisoras de Emergencias, con apoyo técnico de la Sociedad Constructora y aporte Comunitario.

\subsection{Núcleos modulares de hormigón armado: Sistemas tipificados}

En el año 1970, el Taller de Arquitectura de la Zona Central de la Sociedad Constructora inicia el diseño de un proyecto modulado estructuralmente en hormigón armado con bloques autónomos de $16,80 \mathrm{~m} \times 17,40 \mathrm{~m}$, conectados entre si directamente o por medio de unidades con circulaciones verticales. Este sistema, que persigue la máxima flexibilidad y versatilidad en el uso de los espacios interiores, que exige la nueva Reforma Educacional implantada en el pais, se utilizó en el Liceo n.o 1 de Valparaiso "Eduardo de la Barra».

La estructura es de tipo tradicional con pilares, vigas y losas con luces máximas de $7,20 \mathrm{~m}$, por razones de factibilidad económica. Sin embargo, se había tomado en cuenta la posibilidad de obtener una estructuración constructiva más avanzada, constituidas por losas nervadas pretensadas y elementos de relleno prefabricados, alternativa que no se llevó a efecto (Lámina IX).

Simultáneamente en la Escuela Normal de la Reina, Santiago, se emplearon estructuras modulares de hormigón armado, agrupadas dentro de una trama racionalizada y orgánica de expansión y una adecuada tipificación de elementos constructivos.

En la actividad privada, ese mismo año 1970, un equipo de Arquitectos también utilizó un sistema parecido, de trama, en el Colegio Saint George de Santiago, en el cual se emplearon núcleos modulares basados en elementos constructivos de hormigón armado, prefabricados y normalizados in situ. Su estructura soportante principal consistía en elementos verticales en cruz y vigas $L$ con sección de $40 \mathrm{~cm}$, de alas, en módulos múltiplos de $3 \mathrm{~m}$ de largo, de hormigón armado vibrado y pretensado. Los elementos de cierre eran móviles y autosoportantes, provistos de aislaciones térmicas y acústicas incorporadas. La estructura de techumbre se consulto en madera predimensionada y tratada, con cubierta de hierro galvanizado emballetado. 


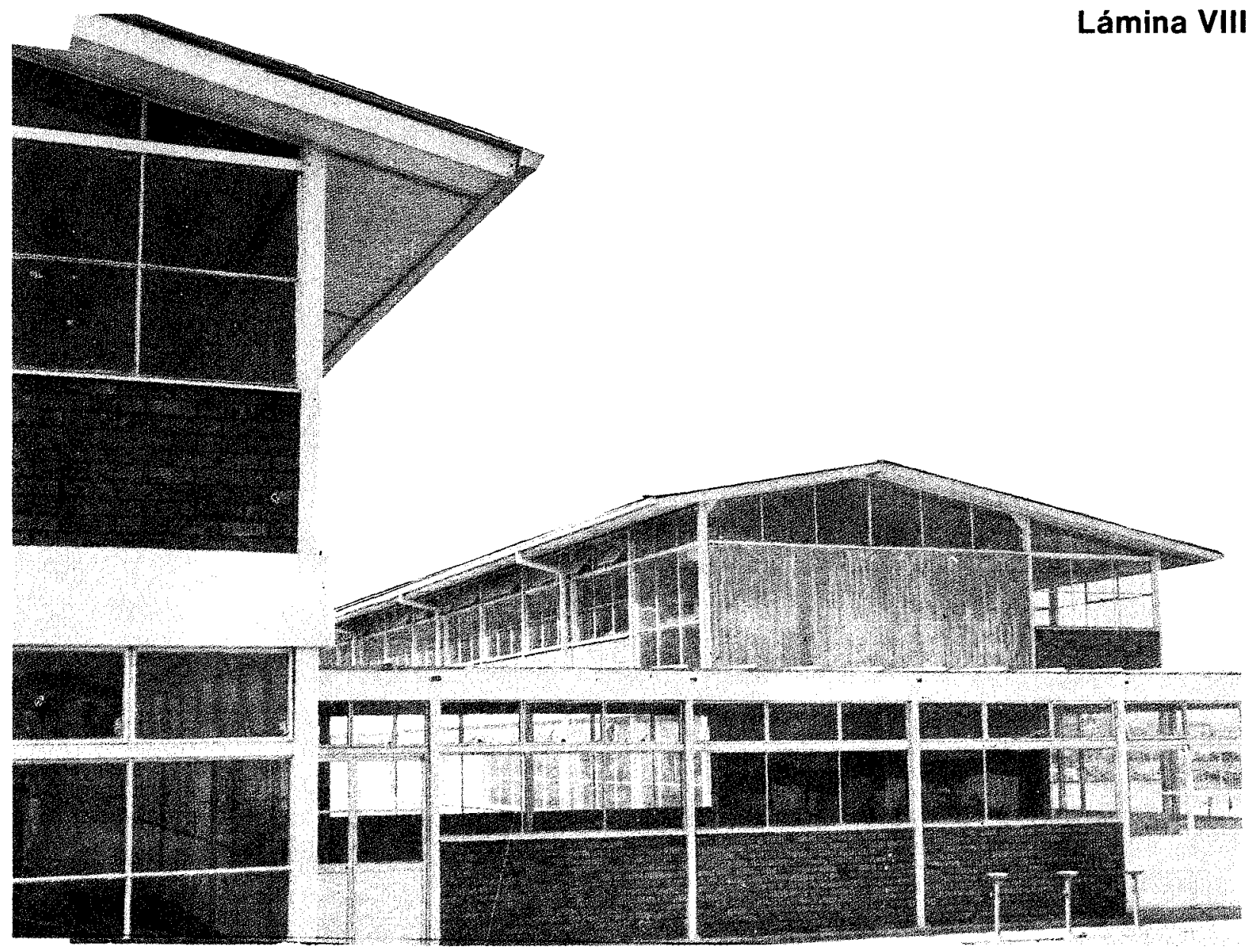

Proyecto tipo 606 - F. Sociedad Constructora de Establecimientos Educacionales.

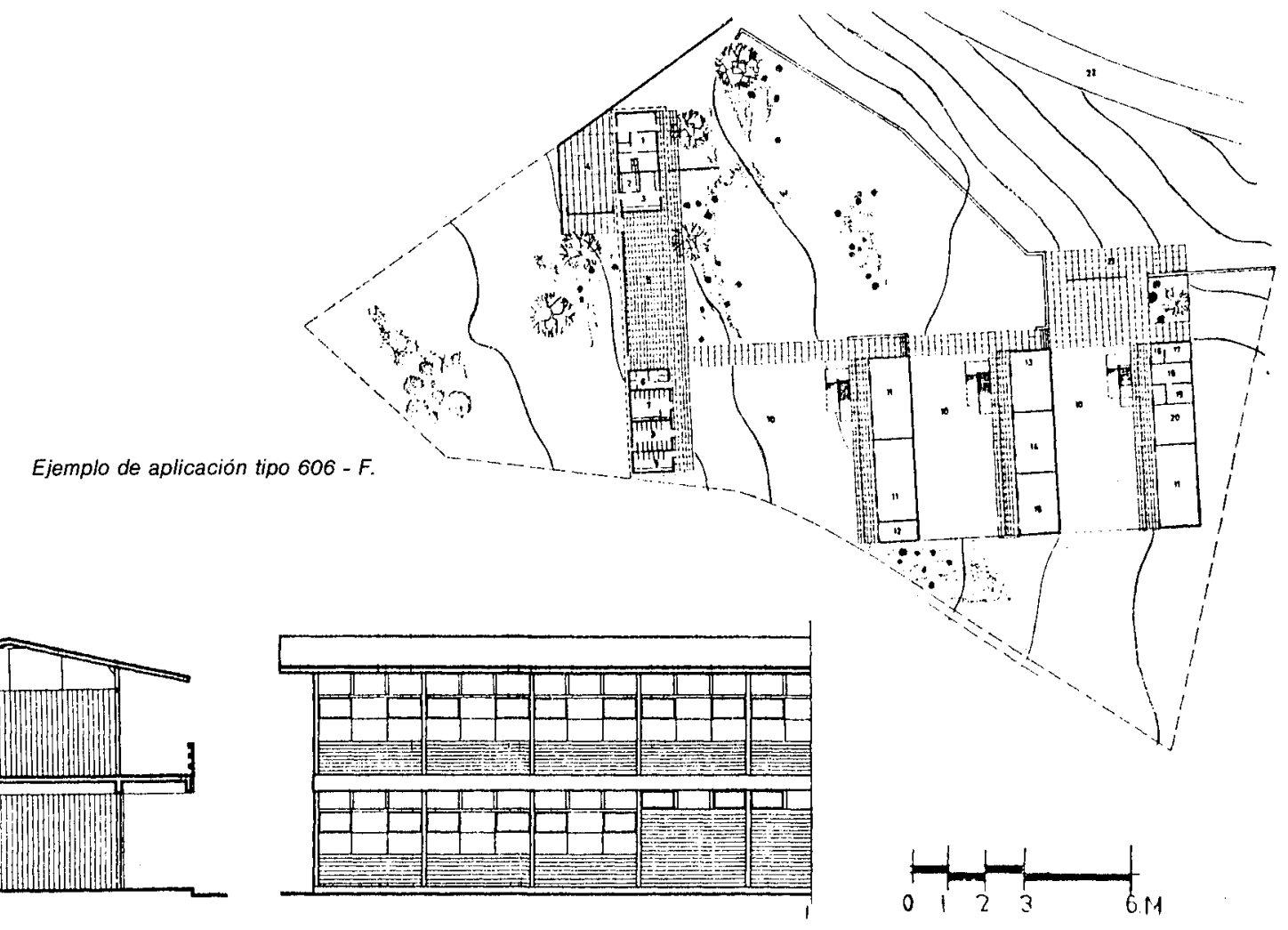




\section{Lámina IX}

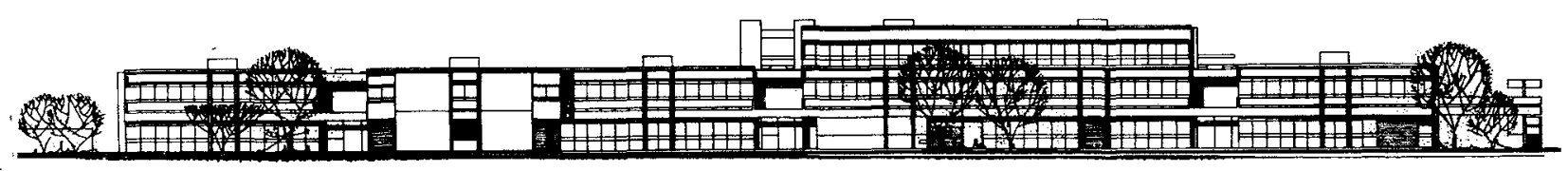

Elevacion general.

Liceo n.o 1. Valparaiso

Taller zona central, 1970

F. Briceño, Arquitecto
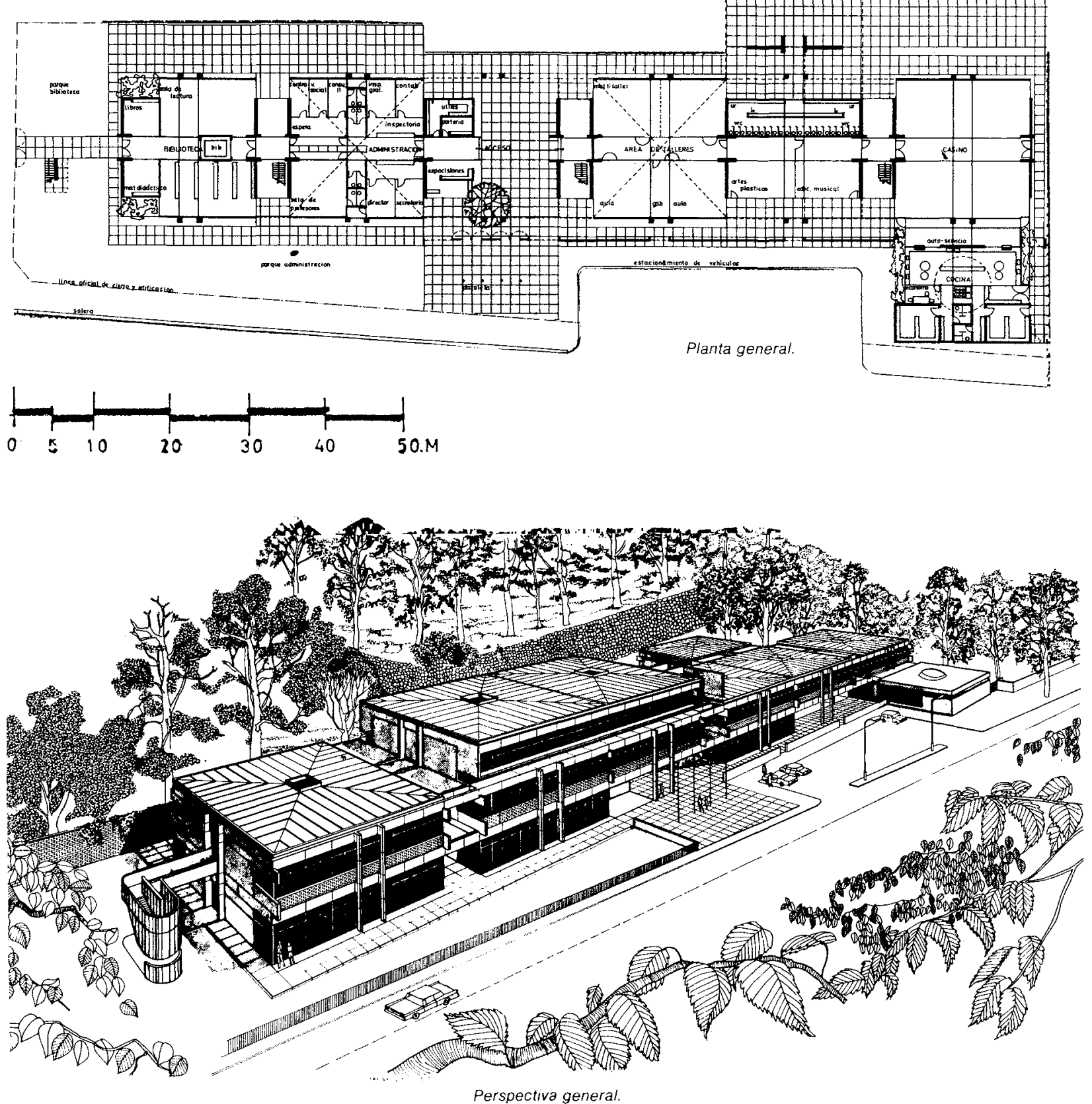
Se da a conocer este ejemplo por considerarlo un aporte singular en la construcción escolar de ese momento (Lámina $X$ ). Asimismo se podria citar un ejemplo similar como el Sistema Constructivo para Edificaciones Educacionales -SCAS - realizado por el Instituto de Desarrollo Experimental de la Construcción de la Facultad de Arquitectura y Urbanismo de la Universidad Central de Venezuela.

Entre los años 1971 y 1972 se realizaron otros proyectos experimentales, en hormigón armado, denominados Tipos 510 y 520 . El primero de ellos, utilizado en el Instituto Comercial de Valparaiso, consistia en adosamientos de megaestructuras, acopladas entre sí o mediante elementos conectores de unión, con circulaciones verticales incorporadas. Estas unidades formadas por marcos rígidos, losas, pilares, y machones de hormigón armado, de 17,50 × 17,50 m, estaban acondicionadas para ser usadas en una, dos y tres plantas y el sub-módulo de diseño era de $1,20 \times 1,20$ metros.

El segundo sistema, que fue empleado en el Instituto Comercial de Viña del Mar, comprendia un módulo generador de $9,00 \times 9,00 \mathrm{~m}$, con un submódulo de diseño de 1,50 $\times 1,50 \mathrm{~m}$ en una, dos y tres plantas, el cual eliminaba los machones de hormigón armado, reemplazándolos por una estructura basada solamente en pilares e incluyendo viguetas y losetas premoldeadas. El cerramiento estaba constituido por paneles prefabricados de acero con revestimientos de asbestocemento, ventanas de perfiles de aluminio con vidrios fijos y elementos de ventilación independientes. En la cubierta se especificaba impermeabilización asfáltica. Este sistema, igual que el anterior, permitía formas de crecimiento bidireccional y obedecia a lograr el máximo de flexibilidad para los espacios docentes interiores.

Se puede mencionar otro ejemplo constituido por un sistema de núcleos modulares que se proyectó en el año 1978, en el Taller de Arquitectura de la Sociedad Constructora, utilizado en el conjunto del edificio destinado a Escuela Industrial de Recursos del Mar, en lquique, cuya primera etapa se realizó ese mismo año (Lámina XI).

Las caracteristicas del emplazamiento correspondian a un lugar norteño próximo al mar, árido y rodeado, en dos costados, por altos cerros sin vegetación.

Se propuso una arquitectura basada en módulos de $7,20 \times 7,20 \mathrm{~m}$, en hormigón armado, insertos en una trama espacial, cuya superficie total seria de $9.800 \mathrm{~m}^{2}$, a desarrollarse en dos etapas. Dichos módulos se consultaron separados por estrechas circulaciones de $2,40 \mathrm{~m}$, a fin de lograr una protección solar en las circulaciones peatonales. Se prescinde del uso de estructuras me- tálicas, por razones de oxidación, y de la madera, por el ataque de insectos.

\subsection{Hormigón armado porticado modulado: Sistemas tipificados}

En los paises del Area Andina, sometidos con frecuencia a fuertes movimientos telúricos destructivos, se tiene especial preocupación en la construcción de edificios con precisas caracteristicas estructurales de comportamiento sismico. De alli la ventaja de utilizar un sistema basado en marcos rígidos de hormigón armado, que permiten dar una respuesta prevista y controlada a los esfuerzos horizontales provenientes de los sismos, dada la relativa flexibilidad de las columnas y la resistencia de las vigas y de los diafragmas que constituyen las losas de entrepisos. A causa de las deformaciones elásticas momentáneas, en los periodos criticos sismicos, se deben tomar las precauciones debidas en las uniones de los elementos rigidos de relleno.

En esta misma calificación se podria considerar, en cierto modo, el proyecto anterior S.T. 520 y algunas obras más que, en forma particular, en la oficina, fueron ejecutadas en Santiago como las Escuelas de Enseñanza Primaria, el Instituto Claudio Matte de Enseñanza Media y el Colegio de los Andes. Todos ellos, en una y dos plantas.

\subsection{Albañilería reforzada modulada: Sistemas tipificados}

En contraste con los sistemas, integramente prefabricados, se realizaron estudios tendentes a utilizar en la construcción un mayor porcentaje de mano de obra en los edificios docentes, de áreas suburbanas y rurales, acudiendo a estructuras tradicionales de albañilería de ladrillos reforzadas, por razones de factibilidad económica.

Entre los prototipos que tuve a mi cargo desarrollar figura el sistema denominado 330 AR, realizado en 1980 para la Escuela Rural Villamávida, y que se han seguido utilizando, en lineas generales, hasta el dia de hoy.

Este consistía en el empleo de albañileria de ladrillo hecho a mano en la región con pilares, vigas y cadenas de hormigón armado, con una techumbre estructurada en perfiles de hierro y costaneras de maderas.

La iluminación bilateral se conseguia mediante la adopción de una cubierta en un sólo sentido, a un agua, lo que permitia, a su vez, una adecuada ventilación transversal de los recintos docentes. Dentro de la misma modalidad, se puede considerar el proyecto que ejecute para la Escuela $R u$ ral de Puangue, que contemplaba el aprovechamiento de antiguas cubiertas de tejas de arcilla, 


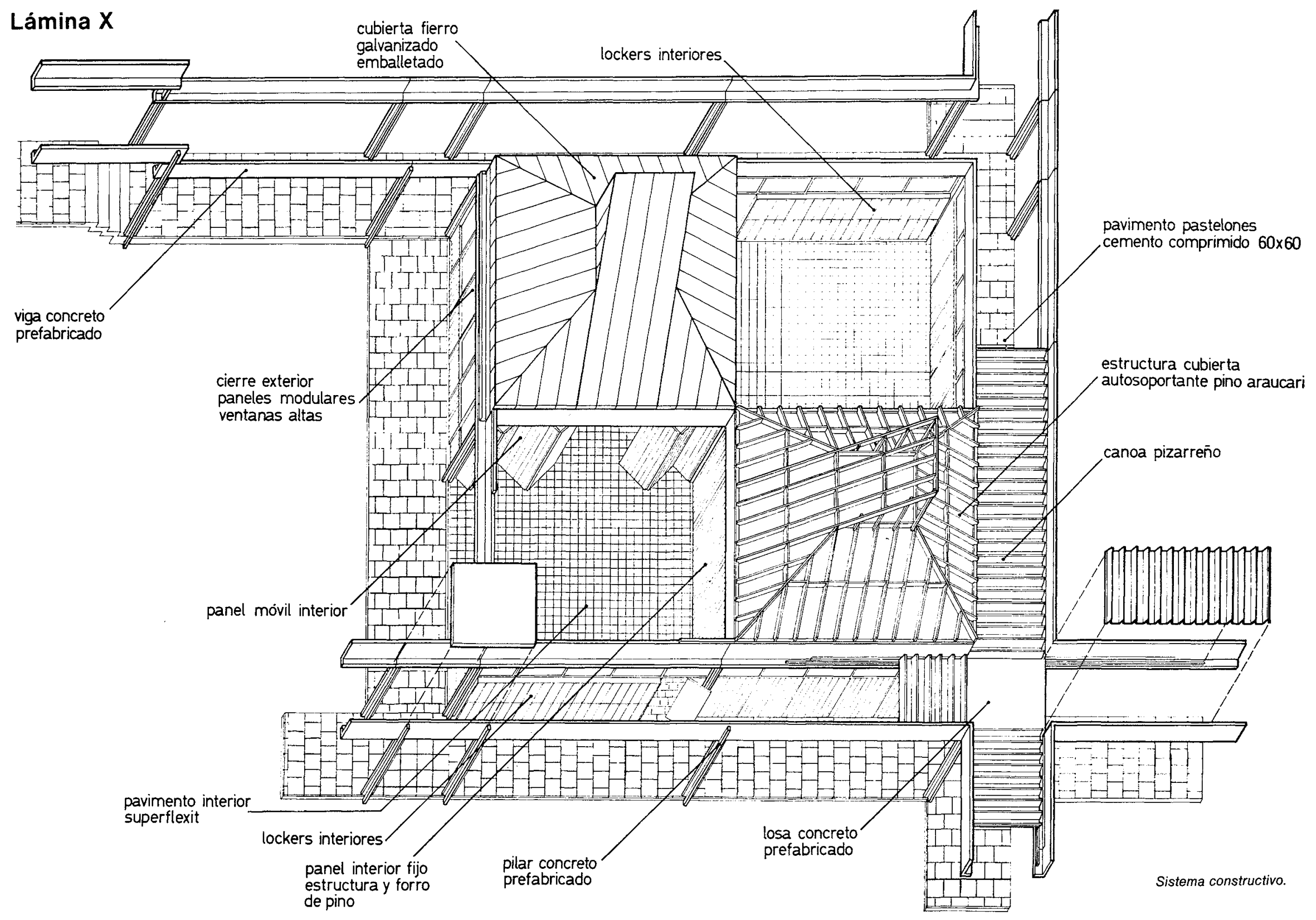




\section{Lámina XI}
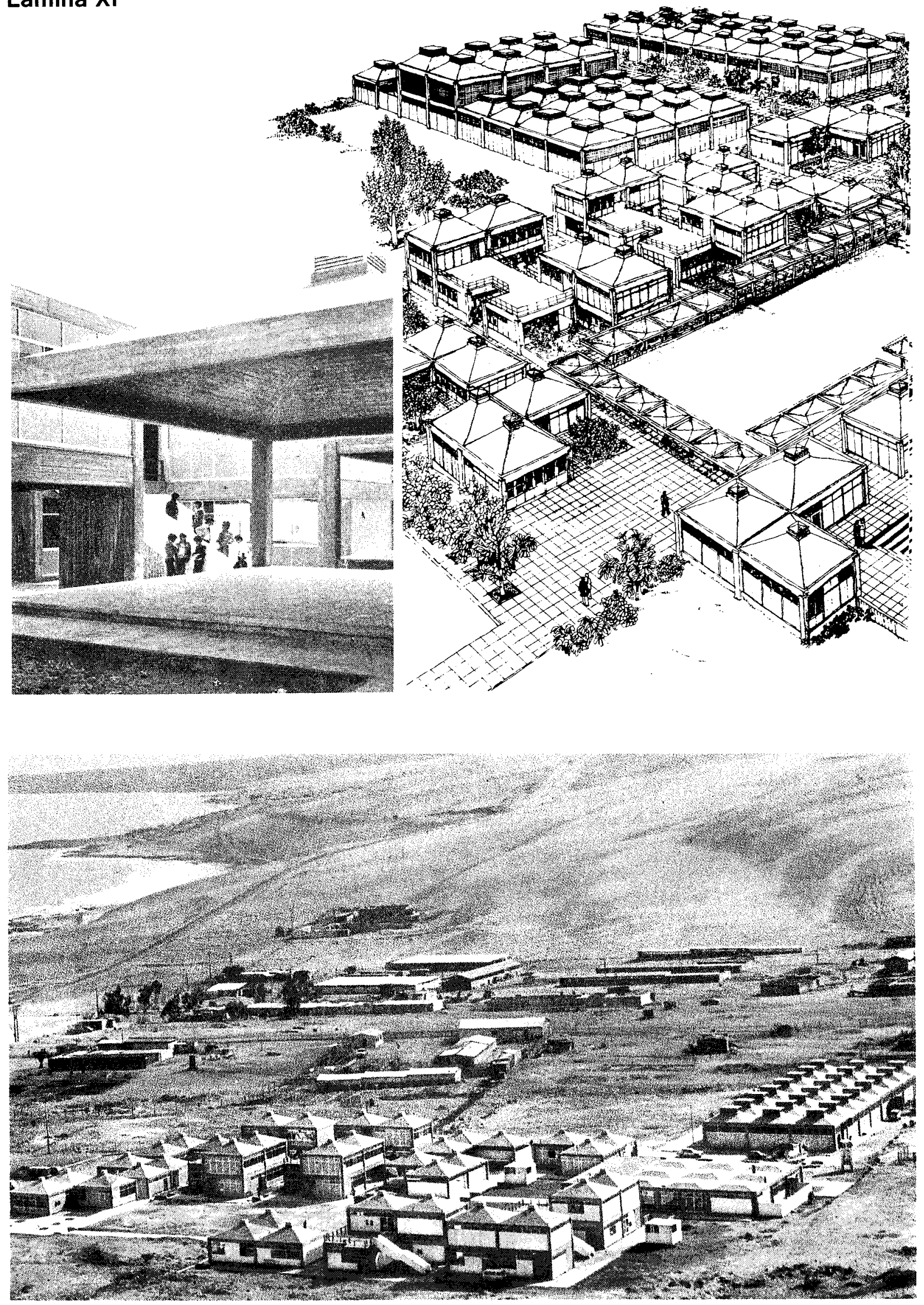

Escuela Industrial de Recursos del Mar. Iquique, Taller Norte. F. Toro, Arquitecto 
piezas de madera, bases de piedras y otros materiales provenientes de la demolición de una construcción existente, en mal estado, de una vieja mansión patronal, en el mismo lugar. Se diseñaron las aulas en torno a un patio central, rodeado de corredores, similar a tradicionales casas solariegas de campo, aún vigentes. Se especificaron muros de albañileria de ladrillo hecho a mano del lugar, con cadenas, vigas y pilares de hormigón armado y cerchas de madera en la techumbre (Lámina XII).

\subsection{Albañilería reforzada y hormigón armado tradicional: Sistema atípico}

Se ha comprobado que las estructuras mixtas compuestas de pilares, vigas y losas de hormigón armado, que consultan muros de albañileria con sus cadenas de hormigón correspondientes, han resistido plenamente en los últimos sismos en el pais y son, por su naturaleza, de menos costos, dada la gran incidencia de mano de obra en su ejecución.

Dicha circunstancia -competitiva en el campo de la educación- ha motivado la posibilidad de recurrir a este sistema constructivo, en reemplazo de la prefabricación. A pesar de un mayor tiempo que requiere su realización permite, sin embargo, reducir el valor del $\mathrm{m}^{2}$ unitario, siempre que no se exceda de la utilización de materiales de terminación y revestimientos. Es por ello que su empleo en proyectos de establecimientos docentes ha sido siempre un recurso constante y se ha prolongado hasta la fecha. Reúne, además, otras ventajas derivadas de «la gran resistencia del ladrillo que, debidamente producido, nos da tensiones de rotura superiores a las de los mejores hormigones".

Basados en este sistema constructivo se pueden citar diversos edificios docentes, en los cuales participé, tanto en el diseño como en la ejecución de los mismos. Entre éstos cabe destacar el Colegio Regina Mundi, la Escuela Normal Santa Teresa, que se detallan más adelante, y diversas obras privadas de edificios singulares relacionados con la docencia.

En todo caso, es útil recordar que el sistema basado en albañileria reforzada y hormigón tradicional tiene el inconveniente de no ser una solución adecuada para emprender masivos programas de construcciones docentes, sino que está más indicado para edificios singulares y escasamente repetitivos.

\section{PROYECTOS ESPECIALES DOCENTES}

Complementando los planes extraordinarios de construcciones escolares, durante el Gobierno del Presidente Frei, el Ministerio de Educación estimó que los estudios, investigaciones y proyectos arquitectónicos que desarrollaba la Sociedad Constructora deberian rebasar los programas docentes y extender su acción a un amplio campo de orden cultural. Esta iniciativa del Ministerio se tradujo en numerosos proyectos llamados "especiales", en muchos de los cuales participé profesionalmente.

Se incluian en esta denominación: Jardines Infantiles, Centros de Recreación y Cultura, Centros para la Juventud, Bibliotecas, Museos, etc.

En dicha categoría puedo mencionar los proyectos que me encomendó la Sociedad Constructora como: el Aula Magna del Liceo Experimental Manuel de Salas, en Santiago, terminada en 1970; el Museo Gabriela Mistral, en Vicuña, obra entregada en 1971, y el Centro de Perfeccionamiento del Magisterio, en Viña del Mar, terminado en 1974, cuyas descripciones se detallarán más adelante.

En el año 1969 se propuso que la Sociedad proyectara también el nuevo edificio para el Ministerio de Educación, de aproximadamente $32.000 \mathrm{~m}^{2}$ de superficie. Su emplazamiento fue simbólicamente fijado frente al Palacio de Gobierno - La Moneda- dada su vital importancia en el contexto de la política general del país. Estaria separado de aquél por una amplia Avenida de edificios públicos existentes. Su finalidad era reunir las diversas oficinas dependientes de esa Secretaria de Estado, que se encontraban dispersas en el radio urbano, complementando otras actividades de orden científico, cultural y artístico. El equipo encargado del proyecto fue integrado por cuatro arquitectos: $M$. Pérez de Arce, J. Benavides, V. Pereda y el suscrito.

El edificio que constaba de Auditorio, Salas de Exposiciones, Libreria, Imprenta, Estudios de Televisión y Cine, Oficinas Generales, Cafeteria, etc., no se pudo materializar por circunstancias politicas y económicas del momento.

\subsection{Aula magna del liceo experimental Manuel de Salas, Santiago}

Este edificio, con una capacidad de 600 butacas, formaba parte de un equipamiento de divulgación cultural que consultaba además Biblioteca, Departamento Audiovisual y Talleres Artísticos. Dicha Aula podria indistintamente funcionar como Sala de Conferencias, de Espectáculos y de Cine, lo cual obligó a resolver forzosamente un espacio interior de uso múltiple, utilizando un sistema acústico especial en el techo y placas absorbentes de ruido en los revestimientos laterales y posteriores. Cerchas metálicas de una altura aproximada de $2,00 \mathrm{~m}$ accesibles salvan la luz de $29 \mathrm{~m}$, entre muros resistentes exteriores. 


\section{Lámina XII}

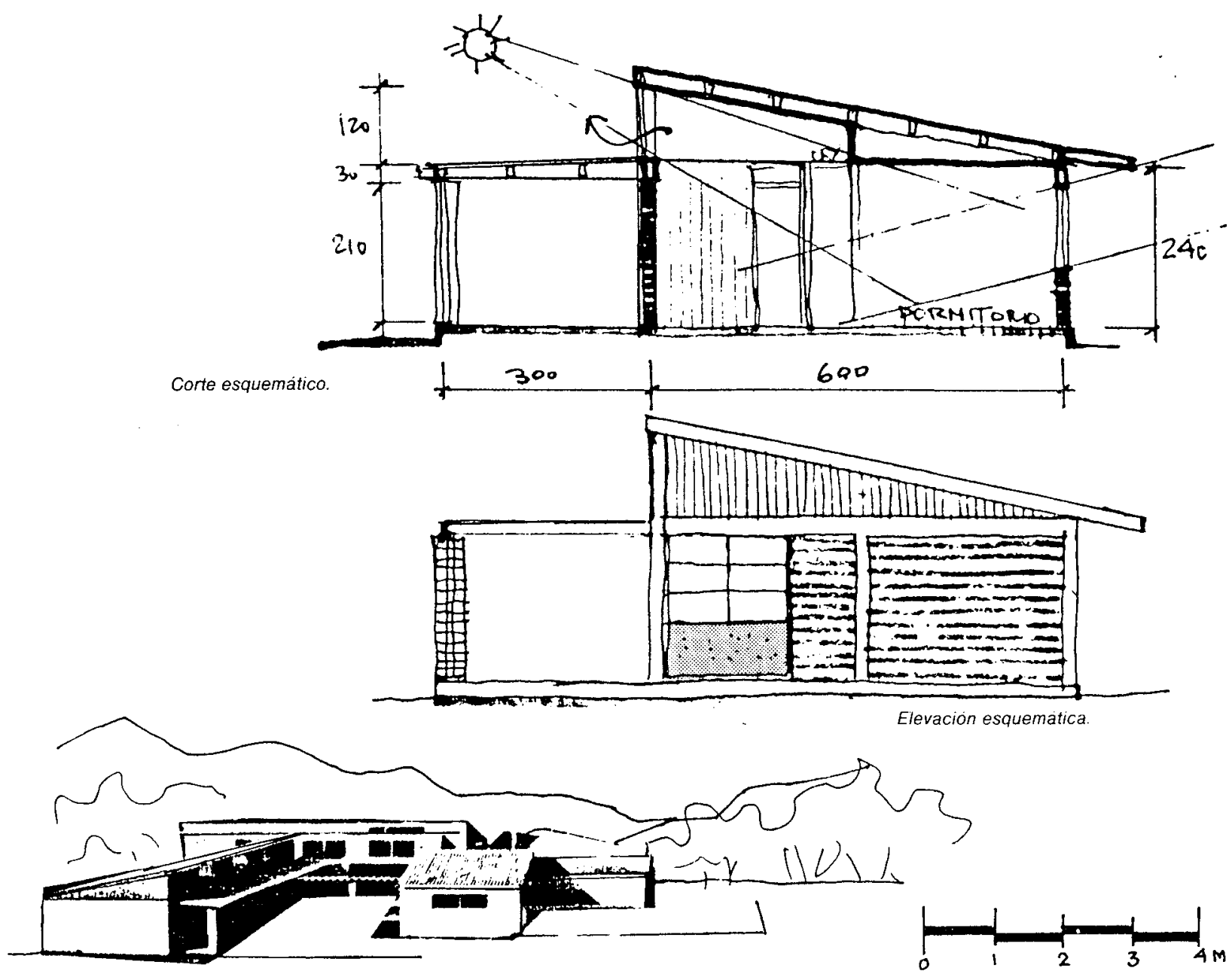

Sistema 330 - AR. Albarileria reforzadt. 1980

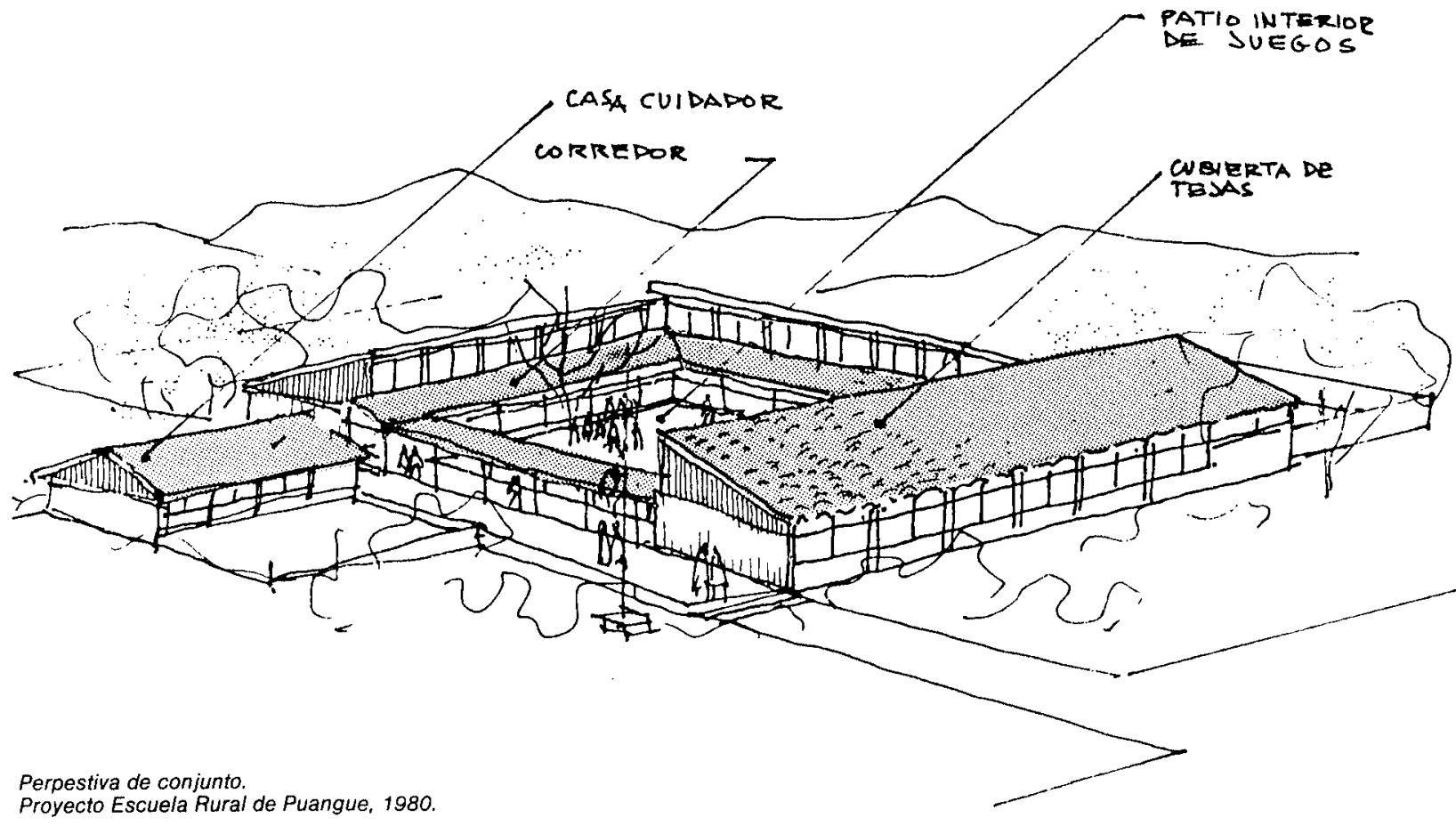


Estos últimos se ejecutaron en albañilería reforzada de ladrillo prensado a la vista.

\subsection{Museo Gabriela Mistral, Vicuña}

Este Museo-Biblioteca, rodeado de parque con árboles autóctonos centenarios, fue erigido en homenaje a la maestra y poetisa Gabriela Mistral -Premio Nobel de Literatura, 1945- y está construido en el mismo lugar de su nacimiento.

El edificio tiene sólo $400 \mathrm{~m}^{2}$ construidos, superficie que se amplia al exterior con patios semi cerrados y terrazas, adecuados al microclima de Vicuña.

Se incorporaron a la obra ciertos materiales caracteristicos del entorno: piedra bolón del río Elqui, de muy variado colorido, se utilizó en los muros del edificio y en los patios adyacentes.

Algunos de estos muros han sido construidos en forma de "pircas", similares a las que construian los incas a lo largo de los caminos. En pavimentos exteriores se colocó cerámica roja de arcilla de $0,25 \times 0,25 \mathrm{~m}$, material utilizado en edificios antiguos coloniales.

El edificio principal de planta cuadrada se cubrió con una losa de hormigón armado, sostenida por cuatro machones estructurales, martelinados. Se construyó una claraboya cilindrica de hormigón, pintada interiormente de color amarillo, y que arroja un haz de luz sobre una jardinera central, en la que se ubicó un cubo de hormigón martelinado con versos de la Poetisa.

Cabe señalar que la limpidez constante del cielo de Vicuña permite que la luz diurna penetre por el cristal de la claraboya e ilumine mágicamente el espacio interior.

Todos los muros de piedra se autosoportan y, estando separados de la estructura del cielo, van conformando y envolviendo diferentes situaciones espaciales interiores.

Hasta la fecha, aún no se ha construido la Sala Audiovisual que complementa el recorrido didáctico del museo.

Esta obra mereció el Premio Anual de Arquitectura, con Mención «Equipamiento", otorgado por el Colegio de Arquitectos en el año 1972.

\subsection{El centro de perfeccionamiento del Magisterio, en Viña del Mar}

Surgió de un Concurso interno convocado por la Sociedad Constructora para construir la nueva sede de la Escuela Normal, cuyo edificio fue destruido por el sismo de 1971, en el mismo lugar. EI proyecto, de una superficie aproximada de 5.000 $\mathrm{m}^{2}$, constaba de tres plantas. En las primeras se situaban las aulas, bibliotecas, administración, casino, laboratorios y talleres. La tercera planta estaba destinada a dormitorios, sala audiovisual y terrazas - jardines.

El conjunto arquitectónico se organizó en torno a dos patios interiores, uno de ellos con tratamiento de jardines y el otro con graderías en torno a un foro, el cual está cubierto por una cúpula piramidal vidriada de estructuras metálicas.

Es una obra compacta, en hormigón armado, que pretendió lograr un nuevo tratamiento arquitectónico espacial en forma concentrada de las áreas docentes.

Este edificio, que no se realizó en su totalidad, sufrió serias transformaciones y omisiones que desvirtuaron ciertos conceptos importantes enunciados en el proyecto. Obtuvo, sin embargo, también el Premio Anual de Arquitectura y Urbanismo, otorgado por el Colegio de Arquitectos el año 1975.

\subsection{Planetario de Santiago}

El Ministerio de Educación, durante el Gobierno del Presidente Frei, prosiguió con su programa de difusión cultural y científica. En 1969, me encargó el proyecto para el Planetario de Santiago, a través de la Sociedad Constructora de Establecimientos Educacionales.

A fin de obtener información técnica al respecto, fui en Comisión de Servicio, ese mismo año, a la Industria Carl Zeiss de Oberkochen, Alemania Occidental, realizando otras visitas programadas a los Planetarios de Nurenberg, Berlin, Zurich, Lucerna, París y Londres. Posteriormente visité por mi cuenta los Planetarios de Nueva York y Buenos Aires. En México visité los del Instituto Politécnico de Zacatenco en la Capital Federal y los de Morelia y Villa Hermosa. Este último de grandes proporciones y muy bien equipado.

El Planetario de Santiago, por distintas circunstancias, se terminó definitivamente el presente año 1986, y está en pleno funcionamiento en terrenos pertenecientes a la Universidad de Santiago (ex Universidad Técnica del Estado).

Dicha obra, de $4.000 \mathrm{~m}^{2}$ de superficie aproximada, también tuvo durante su ejecución diversas modificaciones que en cierta manera afectaron al concepto inicial del proyecto realizado.

No se ha llevado a cabo, hasta el momento, el acceso principal peatonal programado, que convergia diagonalmente con un Anfiteatro al aire libre, donde se habia propuesto instalar un gran reloj de sol metálico de $5 \mathrm{~m}$ de altura y una fuente de agua ornamental. 


\section{CONSTRUCCIONES DOCENTES PRIVADAS}

A) Asociado con el Arquitecto Hernán Mönckeberg B., Presidente de la Sociedad Constructora de Establecimientos Educacionales, entre los años 1975 a 1978, nos fueron encargados los Centros Culturales de la Caja de Compensación de la Cámara Chilena de la Construcción, en Santiago y Concepción de $6.000 \mathrm{~m}^{2}$ y $8.000 \mathrm{~m}^{2}$ respectivamente. Las dos obras obtuvieron el primer premio con Mención «Equipamiento", seleccionadas por el Colegio de Arquitectos de Chile, entre las mejores obras construidas aquellos años en el pais. En ambos edificios se consultaban zonas de oficinas, talleres, salas de exposiciones, teatros, bibliotecas, auditorios y cafeteria.

El edificio de Santiago incluye una piscina deportiva y un gimnasio cubierto, con graderías en dos plantas. Actualmente, éste se utiliza como espacio de uso múltiple, para basquet-ball, conciertos, espectáculos de ballet, presentaciones de grupos folklóricos, etc.

También, asociado con el arquitecto Mönckeberg, realice diversas obras de carácter docente como Escuelas de Enseñanza Primaria, Instituto Claudio Matte de Enseñanza Media, en Santiago y el Colegio Seminario San Rafael de Valparaiso.

En cada uno de ellos se aplicaron nuevas experiencias, tanto en aspectos arquitectónicos como constructivos, logrando disminuir notoriamente los costos normales en este tipo de construcciones docentes.

B) En forma particular me fueron encomendados otros establecimientos de Enseñanza Básica, Media y Normal.

- El más antiguo, en los años 1960, fue la Escuela de la Congregación de las Hermanas de la Caridad, proyecto que se construyó en terrenos vecinos al Seminario Vicentino, también iniciado en esos años. Las dos obras se realizaron con gran economia de medios.

- Proyecto complejo fue el encargado por la Institución Teresiana, que abarcaba los tres estamentos: Enseñanza Básica, Media y Normal. En un principio se denominó "Escuela Normal Santa Teresa", hasta 1974, año que fueron suprimidas todas las Escuelas Normales del pais. Este trabajo habia. sido encomendado por la Institución al Arquitecto español Manuel Barbero Rebolledo, en Madrid. El vino a Chile, en esa oportunidad, y empezamos juntos a trazar los primeros bosquejos del anteproyecto La superficie inicial de este conjunto educacional era de $20.000 \mathrm{~m}^{2}$, de los cuales se han ejecutado hasta la fecha sólo $5.000 \mathrm{~m}^{2}$.

- El Colegio Tabancura, perteneciente a la Sociedad de Educación (SEDUC) del Opus Dei, contemplaba los estamentos de Educación Básica y Media. En el conjunto del edificio, de una superficie aproximada de $4.000 \mathrm{~m}^{2}$, construidos, se lograron ciertos aportes arquitectónicos y constructivos, basados en una estructura metálica similar al proyecto tipo 720 de la Sociedad Constructora de Establecimientos Educacionales (S.C.E.E.).

- El Colegio de Los Andes, también de SEDUC, con una superficie equivalente al anterior, está constituido por edificios de una y dos plantas en hormigón armado, con estructura soportante porticada en base a pilares de $0,40 \mathrm{~m} \times 0,40$ $m$ y elementos de cierre, en albañileria de ladrillos a la vista y madera.

\section{PROGRAMAS DE ACCION}

Considero necesario señalar el impetu que, como politica fundamental de construcciones docentes, le imprimieron los últimos Gobiernos Democráticos correspondientes a los Presidentes Alessandri, Frei y Allende, entre los años 1959 y 1973.

Ese vigoroso impulso se encauzó hacia la realización material de planes anuales destinados a solucionar, a corto y mediano plazo, el déficit de Establecimientos Educacionales en todo el pais. El mayor énfasis estuvo radicalizado en sectores urbanos, ya que ellos representan el $70 \%$ aproximadamente de la población nacional.

Se estimó que la actividad privada estaba limitada a realizar solamente algunos proyectos específicos de Escuelas, Institutos y Colegios particulares. Pero la Construcción masiva de edificios docentes fiscales deberia estar a cargo de una Institución Estatal que pudiese responder con prontitud a la demanda escolar, como era la Sociedad Constructora de Establecimientos Educacionales, dependiente del Ministerio de Educación Pública.

Para llevar a cabo dichos planes anuales, normales y extraordinarios, de edificación docente, fue necesario organizar una acción operativa y reforzar adecuadamente el equipo profesional interdisciplinario existente en esa Sociedad Constructora.

De ese modo, se fueron perfeccionando tècnicamente los sistemas constructivos y proyectos arquitectónicos de carácter docente, introduciendo la prefabricación como un camino más viable para resolver, oportunamente, las exigencias en aumento de la demanda educacional.

Como consecuencia de este ritmo acelerado de construcciones escolares se produjo, probablemente, un deterioro en la calidad arquitectónica de los edificios, en pro de soluciones cuantitativas y más rápidas de ejecutar. 
A fin de lograr una diferenciación en el empleo de materiales apropiados a cada localidad del pais, $y$ de obtener una eficiente fiscalización técnica y administrativa de los edificios escolares, se organizaron, en la Sociedad Constructora, Oficinas Zonales con atribuciones propias de control de obras, ubicadas en ciudades representativas del territorio nacional. En Santiago se mantuvo una central de programación de operaciones y financiamiento de los planes a realizar. El taller de Arquitectura se subdividió, a su vez, con el mismo objeto, creándose sub-talleres especializados de las distintas zonas: Norte, Central, Metropolitana, Sur y Austral, que analizaban separadamente las condiciones climáticas y geográficas locales a fin de dar respuestas arquitectónicas adecuadas para cada región.

Es preciso reconocer que el éxito logrado se debió, en gran parte, al espíritu de sacrificio, mistica, y entusiasmo del equipo interdisciplinario integrado por Arquitectos, Ingenieros Estructurales, Constructores Civiles y Técnicos en general.

Con ello se logró sobrepasar, en aquel periodo del «boom» de la edificación escolar, durante los planes masivos extraordinarios de edificación comprendidos entre los años 1968 y 1971, la cifra "record" de $200.000 \mathrm{~m}^{2}$ anuales (1).

En forma paralela, e independiente, en la Sociedad Constructora se creó un grupo de profesionales, del cual formé parte integrante, destinado con exclusividad a la investigación y experimentación de nuevos sistemas constructivos y para elaborar proyectos especiales, los cuales requerirán un mayor plazo de desarrollo.

\section{PROGRAMAS DE INTERCAMBIO}

Me parece oportuno recordar que una buena parte de la fructífera labor desempeñada, en la realización de proyectos educacionales, se debió a que gran número de profesionales tuvimos la oportunidad de perfeccionar nuestro quehacer mediante el intercambio profesional y tecnológico con otros paises.

Es asi como en 1967, asisti por dos meses a México D. F., como Arquitecto Delegado de Chile, al Tercer Curso Intensivo sobre Planeamiento, Diseño y Ejecución de Construcciones Escolares para América Latina, realizado por CONESCAL (Centro Regional de Construcciones Escolares para América Latina) (2). Dicho Organismo internacional y autónomo fue creado por la UNESCO y el Gobierno de México, en 1963, y fue patrocinado por la OEA y ese Gobierno.

El Centro estaba dedicado fundamentalmente a la investigación en el campo de las construcciones escolares y, además, colaboraba prestando asesorias técnicas docentes a los Gobiernos de América Latina que to requerian. Por desgracia, actualmente, ya terminó sus labores.
En esa ocasion se visitaron Escuelas construidas por el Comité Administrador del Programa Federal de Construcciones de Escuelas de México CAPFCE (3). El mencionado Organismo creado en 1944, por el Gobierno Mexicano, fue una eficiente Institución que dio respuesta a la imperiosa necesidad de construir edificios escolares adecuados para todo el amplio territorio de México.

El sistema empleado por el CAPFCE consistia en una estructura metálica modulada y tipificada que podía ser utilizada en una y dos plantas. Dicho sistema sirvió de base para los proyectos tipificados utilizados masivamente por la Sociedad Constructora de Establecimientos Educacionales, hasta el dia de hoy.

Anualmente se realizaban estos Cursos donde se reunian Arquitectos, Ingenieros, Pedagogos, Economistas y Sociólogos. Ello facilitaba el intercambio de ideas y prácticas profesionales, a la vez que generaban, para el futuro, lazos de auténtica amistad. Fruto de esta última fueron las visitas que años siguientes realicé a Argentina, donde tuve la ocasión de conocer diversos edificios educacionales realizados por Arquitectos pertenecientes, directa o indirectamente, a la Secretaría de Estado de Cultura y Educación, ubicados en Buenos Aires, Morón, La Plata, Córdoba, Mar del Plata y Mendoza (4).

Los proyectos y obras que visité utilizaban en gran medida el hormigón armado, constituido por marcos rigidos porticados, incorporado a soluciones concentradas, en su mayoría, en esquemas de monobloques de dos o más plantas, modulados y tipificados. Hay que hacer la salvedad de que, en la región próxima al Atlántico, no hay movimientos sísmicos, lo que facilitaba la esbeltez de los componentes estructurales.

Entre los edificios visitados, uno de los más interesantes probablemente, era el Colegio Nacional de Morón, en la Provincia de Buenos Aires, obra del grupo IRA (5), que consta de cuatro plantas con agrupación de aulas en torno a un espacio central balconeado y terraza superior de recreo.

También me parece notorio recalcar que la Dirección Nacional de Arquitectura Educacional de Argentina contemplaba en sus planes la ejecución de otros edificios afines de carácter cultural -en forma similar a nuestro país-como el PabeIlón de esa Nación, en la Ciudad Universitaria de Madrid, la Biblioteca Nacional, Teatro Nacional Cervantes, etc.

Convenios de intercambio con el CAPFCE y CONESCAL, antes citados, permitieron a la Sociedad Constructora que un cierto número de funcionarios y profesionales cualificados ampliaran sus experiencias relacionadas con las nuevas soluciones pedagógicas de orden técnico, aplicadas en lberoamérica. También hubo contactos 
directos con Arquitectos y Pedagogos ingleses, a través del Consejo Británico. Vinieron, en el año 1968, delegados de toda América y de Gran Bretaña a un Seminario sobre Arquitectura Escolar, patrocinado por CONESCAL y realizado por el Centro de Perfeccionamiento e Investigaciones Pedagógicas de Barnechea, Santiago.

A dicho evento concurrieron representantes de 21 paises, integrados por conocidos y prestigiosos profesionales y correspondió al Primer Seminario realizado fuera de México, como homenaje a Chile por la magnitud de las realizaciones hechas por el Gobierno del Presidente Frei en el campo educativo.

La confrontación de experiencias mutuas, efectuadas en los distintos paises, tuvieron singular valor en el diseño de nuevos proyectos educacionales.

Las observaciones señaladas y los contactos profesionales, ya indicados, acentuaron la inquietud por romper con los esquemas tradicionales de crecimiento lineal y de formaciones de bloques paralelos en los diseños, sustituyéndolos por nuevos conceptos de multidireccionalidad, como los que ya se experimentaban en Gran Bretaña y Estados Unidos. Estos antecedentes, sirvieron de modelo para la ejecución de algunos proyectos experimentales realizados en el pais.

Documentos Técnicos relativos a sistemas de prefabricación, como el denominado CLASP, de estructura metálica, fueron proporcionados, gentilmente al Taller de Arquitectura de la Sociedad Constructora por los observadores ingleses del Consejo Británico, que habian asistido al Tercer Seminario Regional de CONESCAL, ya citado. Dicho sistema, como también el S.C.S.D. (6), u otros similares, persiguen utilizar elementos metálicos predimensionados de pilares y vigas estructurales, en una y dos plantas y que, como mecano, se pueden adaptar a distintas situaciones derivadas de necesidades pedagógicas.

En otros sistemas constructivos de interés, en el campo de la prefabricación de edificios educacionales, cabe señalar el denominado SERP (7), utilizado en Escuelas Rurales del Perú, basado en estructuras metálicas livianas de fácil transporte y armado. También se puede mencionar el Sistema SCAP (8), en hormigón armado, propuesto por el Instituto de Desarrollo Experimental de la Construcción, en Venezuela.

\section{PROGRAMAS ACTUALES}

No podriamos dar por terminado este trabajo, que es como una recopilación cronológica del quehacer arquitectónico en torno a la actividad docente y cultural, sin antes referirnos a la situa- ción actual de la Sociedad Constructora de Establecimientos Educacionales.

Para establecer la magnitud en materia de construcciones escolares, me ha parecido necesario hacer un análisis comparativo de la labor reciente de esa Sociedad con respecto a la actividad desarrollada años atrás.

En conformidad con los datos estadisticos recogidos en las Memorias Anuales del Directorio de esa Sociedad (9), se ha podido establecer una notable disminución de superficie edificada de obras entregadas al uso, realizadas en los últimos cinco años (Lámina XIII). También se puede constatar que en ese período no sólo disminuyó la labor de infraestructura escolar y de su necesaria proyección cultural, sino que se detuvo el proceso de experimentación e investigación docente. Tampoco se avanzó mayormente en el diseño arquitectónico, utilizando en general los mismos proyectos tipificados que se habian empleado en años anteriores, con leves modificaciones.

Aquello refleja, en gran medida, el ostensible deterioro sufrido como recesión general de actividades del país en el campo de realización de obras, acentuado por la nueva política educacional implantada por el actual Gobierno tendente a la privatización de la enseñanza.

Actualmente la Sociedad Constructora se ha convertido sólo en una Unidad Técnica, operativa y administrativa, que realiza convenios especificos con las Municipalidades e Intendencias, ofreciendo sus servicios remunerados en base a un listado definido de prioridades, alcanzando sólo apenas la cifra de $40.000 \mathrm{~m}^{2}$, anuales construidos (Lámina XIII).

\section{CONCLUSIONES}

El hecho ya señalado de haber logrado concretar, en su momento, los ambiciosos planes programados de construcciones escolares, como respuesta ineludible a las inquietudes del Magisterio, le significó al pais, durante el periodo comprendido entre los años 1959 y 1973, un prestigio bien merecido a nivel nacional $e$ internacional.

Contrasta aquella entusiasta politica educativa con la sustentada por el actual Gobierno militar que, por Decreto Ley 1-3063 del 13/6/80, traspasa a las Municipalidades, Escuelas y Liceos Fiscales. Conjunto con estas medidas, encarga también a las Municipalidades la administración y ejecución de los edificios escolares, organismos que no cuentan con profesionales especializados en este tipo de construcciones. 


\section{Lámina XIII}
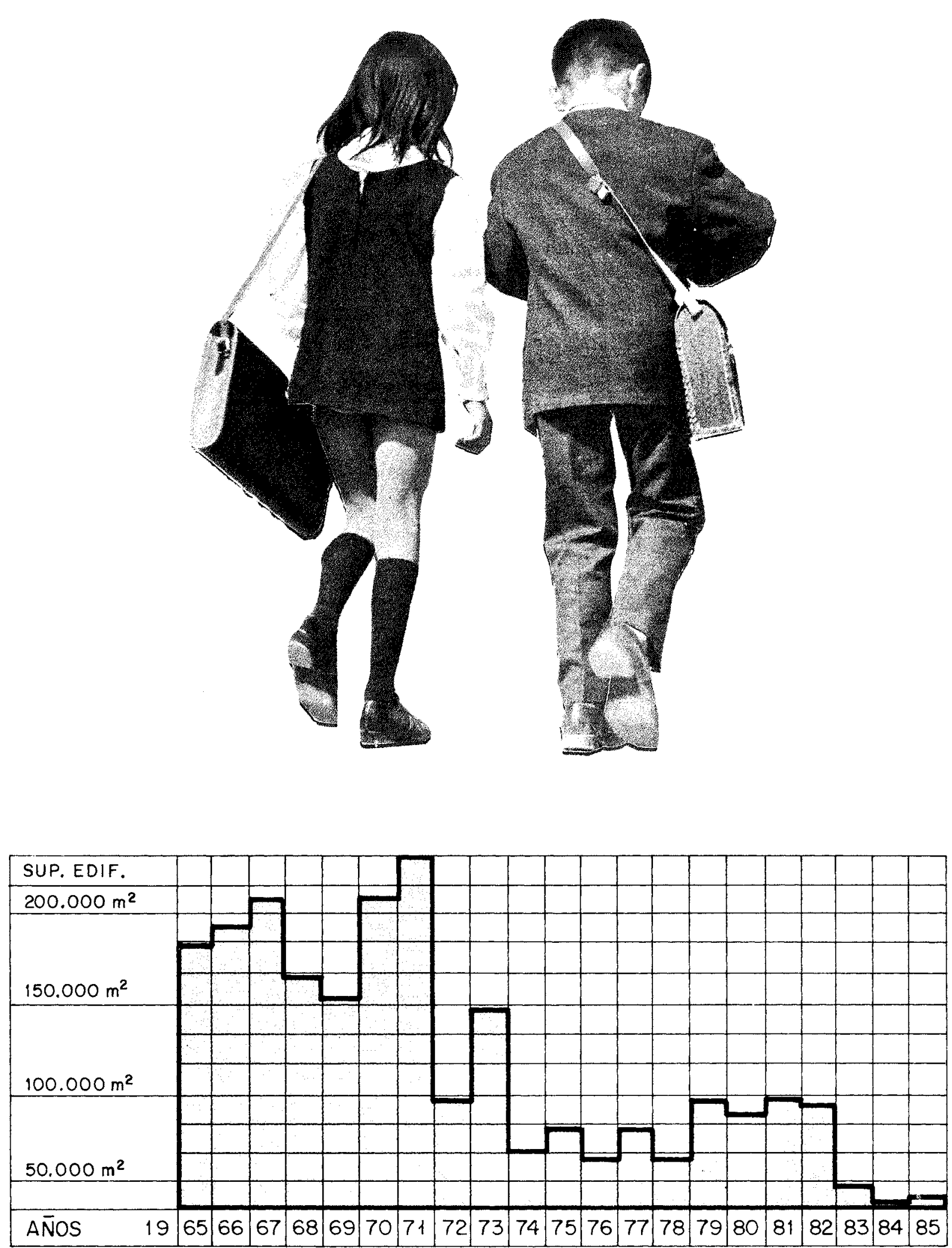

Grafico de obras ejecutadas por la Sociedad Constructora de Establecimientos Educacionales. 
Esta situación se dificulta aún más al considerar que los Alcaldes Municipales son denominados por el Presidente de la República, lo cual significa que no ejercen su cargo por un determinado periodo de años, sino mientras cuentan con la confianza del Presidente.

Asi, es difícil que estos funcionarios públicos -que generalmente no son especialistas en las materias que atañen a la enseñanza y las construcciones escolares- puedan crear y dirigir en el futuro una política de construcciones escolares, técnica y eficiente para el corto, mediano y largo plazo.

Consecuente el Gobierno con su politica educacional, y de acuerdo con la Ley 7689 , el próximo año 1987 se pondrá término a las funciones de la Sociedad Constructora de Establecimientos Educacionales que, como se ha expresado, logró llevar a feliz término en su momento los planes masivos de edificaciones docentes que proponia el Ministerio de Educación Pública.

Queda la esperanza de que, en un futuro próximo, las politicas educacionales del país reconsideren las medidas adoptadas en estos últimos años, que ya han sido expuestas.

También es posible conjeturar que la tarea de Arquitecturas y Construcciones docentes vuelva a su cauce, que se vio interrumpido cuando sus esfuerzos estaban logrando en gran medida alcanzar las metas propuestas en su campo de acción.

Todo logro, toda meta, es posible de ser superada. En consecuencia se espera que, en breve, puedan entregarse "soluciones actuales" válidas para los problemas de las construcciones docentes en Chile, y más acordes con el nuevo espíritu que sopla sobre los pueblos de habla hispana.
Llegará el momento, entonces, de revisar con actitud critica lo realizado y experimentado hasta la fecha, en materia de edificios docentes en el país -como las ya expuestas en este trabajo- y proponer en adelante nuevos métodos constructivos y nuevos planteamientos arquitectónicos, basados en técnicas y materiales, más de acuerdo con los tiempos actuales y venideros.

\section{BIBLIOGRAFIA}

1. MAC LURE: Panorama de la Arquitectura Educacional en el último decenio, Revista $A U C A$, n.o 32 , Chile, O./1977, p. 25.

2. ANONIMO: Editorial Revista CONESCAL, n. 042 , México, 1976, pp. 3.

3. FLORES COSIO, A.: La racionalización de los proyectos arquitectónicos, experiencias del CAPFCE. Revista CONESCAL , n.o 39, México, 1976 , pp. 4 a 28.

4. STORNI, ADOLFO, E.: Arquitectura Educacional; una especialidad profesional, Revista SUMMA n. 95, Argentina, 1975, pp. 17 y 18.

5. Grupo IRA: Colegio Nacional de Morón. Separata de la Revista SUMMA n.0 14, Argentina, 1978, pp. 36 a 39.

6. BENET, J.: The First S.C.S.D. School/Publicacion S.C.S.D.: The Proyect and the Schools, USA, 1967, p. 13

7. MINISTERIO DE EDUCACION: SERP: Sistemas de Escuelas Rurales, Perú, 1973, pp. 1 a 38.

8. FACULTAD DE ARQUITECTURA Y URBANISMO: 1976 SCAC: Sistema constructivo para Edificaciones Educacionales, Venezuela, pp. 1 a 9.

9. SCEE: Memorias Anuales de la Sociedad Constructora de Establecimientos Educacionales, Chile, 1964 a 1985.

\section{Fe de errores}

En el n. 0385 de esta Revista, en el articulo "La arquitectura popular como base de una arquitectura bioclimática. Aplicación al enfriamiento pasivo», en la pág. 65, primera fórmula de la segunda columna, se ha deslizado un lamentable error:

dice $\frac{P}{\omega}+\frac{V^{2}}{2 g}+z=($ diferencial $e)$

debe decir $\frac{\mathrm{P}}{\omega}+\frac{\mathrm{v}^{2}}{2 \mathrm{~g}}+\mathrm{z}=\mathrm{cte}$ 